\title{
It Takes Two Planets in Resonance to Tango around K2-146
}

\author{
Kristine W. F. Lam ${ }^{1}$ (1) , Judith Korth ${ }^{2}$, Kento Masuda ${ }^{3,35}$ (1) , Szilárd Csizmadia ${ }^{4}$, Philipp Eigmüller ${ }^{4}(\mathbb{1}$,
}

Gumundur Kári Stefánsson ${ }^{5}$ (1), Michael Endl ${ }^{6}$ (1) , Simon Albrecht $^{7}$, Paul Robertson ${ }^{5}$ (i), Rafael Luque ${ }^{8,9}$, John H. Livingston ${ }^{10}{ }^{(1)}$, Teruyuki Hirano $^{11}{ }^{1}$, Roi Alonso Sobrino ${ }^{8,9}$, Oscar Barragán ${ }^{12}$ (i), Juan Cabrera ${ }^{13}$ (i), Ilaria Carleo ${ }^{14}$, Alexander Chaushev ${ }^{1}$, William D. Cochran ${ }^{6}$ (1) , Fei Dai ${ }^{15,16}{ }^{(10}$, Jerome de $\operatorname{Leon}^{10}$, Hans J. Deeg ${ }^{8,9}$ (1), Anders Erikson ${ }^{13}$, Massimiliano Esposito ${ }^{17}$, Malcolm Fridlund ${ }^{18,19}$ (1) , Akihiko Fukui ${ }^{20}$, Davide Gandolfi ${ }^{21}{ }^{(10}$, Iskra Georgieva ${ }^{18}$, Lucá Gonzalez Cuesta ${ }^{8,9}$, Sascha Grziwa ${ }^{2}$ (1), Eike W. Guenther ${ }^{17}$, Artie P. Hatzes ${ }^{17}$, Diego Hidalgo ${ }^{8,9}$, Maria Hjorth ${ }^{7}$, Petr Kabath ${ }^{22}$ (10), Emil Knudstrup ${ }^{7}$, Mikkel N. Lund ${ }^{7}$, Suvrath Mahadevan ${ }^{23,24,25}$ (1) , Savita Mathur ${ }^{8,9}$ (1) , Pilar Montañes Rodríguez ${ }^{8,9}$, Felipe Murgas ${ }^{8,9}$, Norio Narita ${ }^{8,20,26,27}$, David Nespral $^{8,9}$, Prajwal Niraula ${ }^{28}$ (1), Enric Palle ${ }^{8,9}$, Martin Pätzold ${ }^{2}$, Carina M. Persson ${ }^{18}$, Jorge Prieto-Arranz ${ }^{8,9}$, Heike Rauer ${ }^{1,13,29}$, Seth Redfield ${ }^{14}$ (i), Ignasi Ribas ${ }^{30,31}$ (i) , Marek Skarka $^{22,32}$ (i), Alexis M. S. Smith ${ }^{13}$, Jan Subjak ${ }^{22,33}$ (i), and Vincent Van Eylen ${ }^{15,34}$ (1)

${ }^{1}$ Center for Astronomy and Astrophysics, Technical University Berlin, Hardenbergstr. 36, D-10623 Berlin, Germany; k.lam@tu-berlin.de

${ }^{2}$ Rheinisches Institut für Umweltforschung an der Universität zu Köln, Aachener Strasse 209, D-50931 Köln Germany

${ }^{3}$ Department of Astrophysical Sciences, Princeton University, Princeton, NJ 08544, USA

${ }_{5}^{4}$ Institute of Planetary Research, German Aerospace Center, Rutherfordstrasse 2, D-12489 Berlin, Germany

${ }^{5}$ Department of Astronomy and Astrophysics, The Pennsylvania State University, University Park, PA, USA

${ }^{6}$ Department of Astronomy and McDonald Observatory, University of Texas at Austin, 2515 Speedway, Austin, TX 78712, USA

${ }^{7}$ Stellar Astrophysics Centre, Department of Physics and Astronomy, Aarhus University, Ny Munkegade 120, DK-8000 Aarhus C, Denmark Instituto de Astrofísica de Canarias (IAC), E-38205 La Laguna, Tenerife, Spain

${ }^{9}$ Departamento de Astrofísica, Universidad de La Laguna (ULL), E-38206 La Laguna, Tenerife, Spain

${ }^{10}$ Department of Astronomy, The University of Tokyo, 7-3-1 Hongo, Bunkyo-ku, Tokyo 113-0033, Japan

${ }^{11}$ Department of Earth and Planetary Sciences, Tokyo Institute of Technology, 2-12-1 Ookayama, Meguro-ku, Tokyo, Japan

${ }^{12}$ Sub-department of Astrophysics, Department of Physics, University of Oxford, Oxford OX1 3RH, UK

${ }^{13}$ Institute of Planetary Research, German Aerospace Center (DLR), Rutherfordstrasse 2, D-12489 Berlin, Germany

${ }^{14}$ Astronomy Department and Van Vleck Observatory, Wesleyan University, Middletown, CT 06459, USA

${ }^{15}$ Department of Astrophysical Sciences, Princeton University, 4 Ivy Lane, Princeton, NJ, 08544, USA

${ }^{16}$ Department of Physics and Kavli Institute for Astrophysics and Space Research, MIT, Cambridge, MA 02139, USA ${ }^{17}$ Thüringer Landessternwarte Tautenburg, D-07778 Tautenburg, Germany

${ }^{18}$ Chalmers University of Technology, Department of Space, Earth and Environment, Onsala Space Observatory, SE-439 92 Onsala, Sweden ${ }^{19}$ Leiden Observatory, University of Leiden, P.O. Box 9513, 2300 RA, Leiden, The Netherlands

${ }^{20}$ National Astronomical Observatory of Japan, NINS, 2-21-1 Osawa, Mitaka, Tokyo 181-8588, Japan ${ }_{21}^{21}$ Dipartimento di Fisica, Universitá di Torino, Via P. Giuria 1, I-10125, Torino, Italy

${ }^{22}$ Astronomical Institute, Czech Academy of Sciences, Fričova 298, 25165, Ondřejov, Czech Republic

${ }^{23}$ Department of Astronomy and Astrophysics, The Pennsylvania State University, 525 Davey Lab, University Park, PA 16802, USA ${ }_{25}^{24}$ Center for Exoplanets and Habitable Worlds, University Park, PA 16802, USA ${ }_{25}^{25}$ Penn State Astrobiology Research Center, University Park, PA 16802, USA ${ }^{26}$ Astrobiology Center, NINS, 2-21-1 Osawa, Mitaka, Tokyo 181-8588, Japan 27 JST, PRESTO, 2-21-1 Osawa, Mitaka, Tokyo 181-8588, Japan

${ }^{28}$ Department of Earth, Atmospheric and Planetary Sciences, MIT, 77 Massachusetts Avenue, Cambridge, MA 02139, USA

${ }^{29}$ Institute of Geological Sciences, FU Berlin, Malteserstr. 74-100, D-12249 Berlin, Germany

${ }^{30}$ Institut de Ciències de l'Espai (ICE, CSIC), Campus UAB, C/de Can Magrans s/n, E-08193 Bellaterra, Spain

${ }^{31}$ Institut d'Estudis Espacials de Catalunya (IEEC), C/Gran Capità 2-4, E-08034 Barcelona, Spain

32 Department of Theoretical Physics and Astrophysics, Masaryk University, Kotlárská 2, 61137 Brno, Czech Republic

${ }^{33}$ Astronomical Institute, Faculty of Mathematics and Physics, Charles University, Ke Karlovu 2027/3, 12116 Prague, Czech Republic

${ }^{34}$ Mullard Space Science Laboratory, University College London, Holmbury St Mary, Dorking, Surrey RH5 6NT, UK Received 2019 July 22; revised 2019 December 21; accepted 2019 December 23; published 2020 February 21

\begin{abstract}
K2-146 is a cool, $0.358 M_{\odot}$ dwarf that was found to host a mini-Neptune with a 2.67 day period. The planet exhibited strong transit timing variations (TTVs) of greater than 30 minutes, indicative of the presence of an additional object in the system. Here we report the discovery of the previously undetected outer planet in the system, K2-146 c, using additional photometric data. K2-146 c was found to have a grazing transit geometry and a 3.97 day period. The outer planet was only significantly detected in the latter $K 2$ campaigns presumably because of precession of its orbital plane. The TTVs of K2-146 b and c were measured using observations spanning a baseline of almost 1200 days. We found strong anti-correlation in the TTVs, suggesting the two planets are gravitationally interacting. Our TTV and transit model analyses revealed that K2-146 b has a radius of $2.25 \pm 0.10 R_{\oplus}$ and a mass of $5.6 \pm 0.7 M_{\oplus}$, whereas K2-146 c has a radius of $2.59_{-0.39}^{+1.81} R_{\oplus}$ and a mass of $7.1 \pm 0.9 M_{\oplus}$. The inner and outer planets likely have moderate eccentricities of $e=0.14 \pm 0.07$ and $0.16 \pm 0.07$, respectively. Long-term numerical integrations of the two-planet orbital solution show that it can be dynamically stable for at least $2 \mathrm{Myr}$. We show that the resonance angles of the planet pair are librating, which may be an indication that K2-146 b and c are in a 3:2 mean motion resonance. The orbital architecture of the system points to a possible convergent migration origin.
\end{abstract}


Unified Astronomy Thesaurus concepts: Exoplanet astronomy (486); Transit photometry (1709); Timing variation methods (1703); Mini Neptunes (1063); Exoplanet systems (484); Photometry (1234); Astrometric exoplanet detection (2130); Transit timing variation method (1710)

Supporting material: data behind figures

\section{Introduction}

The Kepler (Borucki et al. 2010) and K2 (Howell et al. 2014) missions have brought about many exciting discoveries since the spacecraft was launched in 2009. Statistical studies using the Kepler planet sample revealed that sub-Neptune size planets with $R_{p}<4 R_{\oplus}$ are by far the most common type of planets in the galaxy (e.g., Borucki et al. 2011; Howard et al. 2012; Batalha et al. 2013; Dressing \& Charbonneau 2013; Fressin et al. 2013; Petigura et al. 2013). Previous works have also shown that short-period planets with radii between 1.5 and $6 R_{\oplus}$ are common in near coplanar multi-planet systems (Lissauer et al. 2011; Fabrycky et al. 2014). More recently, Weiss et al. (2018) used precisely determined stellar and planetary parameters to show that multi-planet systems are dynamically packed and that adjacent planets in the same system are likely to have similar sizes. Millholland et al. (2017) showed that planets in the same system would have similar masses and radii using a sample of uniformly derived planet masses from TTV analysis.

Multi-planet systems are of particular interest because these systems can provide insights about the formation and evolution of our own solar system. Gaining more knowledge about these systems is important for understanding the planetary system dynamics. To address these topics we need to know the planetary parameters, in particular their radii and masses. Unfortunately, only a small number of planets with precisely measured masses are known because there are inadequate telescope resources for sufficient spectroscopic measurements, or the stars are simply too faint. The mass determination for Earthor Neptune-sized planets around Sun-like stars or faint stars by radial velocity $(\mathrm{RV})$ is particularly difficult with currently available telescopes and instrumental techniques because of the small Doppler reflex motion of the host star.

In multi-planet systems, planets can experience mutual gravitational interactions that perturb their orbits. One of the consequences of these is that individual transits vary periodically around a mean orbital period. This effect is referred to as transit timing variations (TTV, e.g., Agol et al. 2005; Holman \& Murray 2005). This effect is most prominent when the orbital periods of the planets are close to a mean motion resonance (MMR), and it can be measured even for low-mass planets. Thus, TTVs are sometimes the only chance to characterize the planetary system. For example, a TTV analysis of KOI-142 revealed a pair of planets orbiting in a near 2:1 resonance (Nesvorný et al. 2013). Extensive RV observations were obtained for K2-19 b and c, a two-planet system in a near 3:2 MMR (Armstrong et al. 2015; Barros et al. 2015; Narita et al. 2015; Nespral et al. 2017). The measured RV masses of the Neptune-sized planets were found to be consistent with the TTV-derived masses. Precise mass determination via TTVs showed a pair of planets, Kepler-36 b and c, that have distinctly different bulk densities, hinting at different formation origins for the planets (Carter et al. 2012). Dynamical modeling of both the TTV and transit duration variations (TDVs) can also reveal mutual inclination of a pair of planets (e.g., Kepler-108; Mills \& Fabrycky 2017) and uncover the presence of an additional non-transiting companion in some cases (e.g., KOI-872 system; Nesvorný 2012, Kepler-448 b and Kepler-693 b; Masuda 2017).

\subsection{The Cool Star K2-146}

K2-146 was first observed in $K 2$ Campaign 5 . The $\sim 2.2 R_{\oplus}$ mini-Neptune, K2-146 b, was validated independently by Hirano et al. (2018) and Livingston et al. (2018). K2-146 b orbits an M3.0V dwarf and was reported to have an orbital period of 2.645 days. The system was also independently flagged as a planetary candidate by Pope et al. (2016), Libralato et al. (2016), and Dressing et al. (2017). Hirano et al. reported strong TTVs with amplitudes of over 30 minutes. The observed orbital perturbation of the planet is likely caused by either a massive object in its vicinity or an additional object orbiting in or close to an MMR. The stellar parameters of K2-146 are summarized in Table 1.

This paper is organized as follows. Section 2 describes the $K 2$ photometric observations, data reduction, and planet detection. Section 3 describes the spectroscopic observations, which are used to rule out the presence of an outer planet companion. Section 4 describes the extraction of transit times for K2-146 b and c, and transit analyses. The TTV model and analysis are described in Section 5. The transit parameters of the mini-Neptune pair are refined following the TTV analysis in Section 6. In Section 7 we evaluate the stability, orbital resonance, and interior composition of the planet paird, and interpret their possible implications for the evolution history of the system. We present our conclusions in Section 8.

\section{K2 Photometry}

K2-146 was observed in Campaigns 5, 16, and 18 (hereafter, $\mathrm{C} 05$, C16, and $\mathrm{C} 18$, respectively) in long-cadence mode. The photometric observations were obtained between 2015 April 27 and 2018 July 2, spanning a baseline of almost 1200 days. The $K 2$ target pixel data were downloaded from the Mikulski Archive for Space Telescope ${ }^{36}$ (MAST). A custom pipeline was implemented for light-curve reduction and is described below.

The photometric analysis was conducted for each campaign separately. For each campaign, the timestamps were combined and the quality of the light curve was tested using different thresholds to the number of counts per pixel. An optimal aperture was selected using the 100 counts per pixel threshold. Using this aperture we calculated the flux for each frame. To correct for possible correlation with the movement of pointing, we cut the light curves into segments with a length of four days. Between adjacent segments, there is an overlapping region of 0.8 days. The overlapping regions help us to avoid edge effects when fitting the data in the time domain to remove stellar variability.

For each light-curve segment, outliers (including transits) were identified and masked before fitting a multidimensional polynomial, over the POS_CORR columns (which measure the

\footnotetext{
${ }^{36}$ http://archive.stsci.edu/kepler/data_search/search.php
} 
Table 1

Stellar Parameters and Photometric Magnitudes of K2-146

\begin{tabular}{|c|c|c|}
\hline Parameter & Value and Uncertainty & Source \\
\hline EPIC & 211924657 & $\mathrm{a}$ \\
\hline 2MASS & 2MASS J08400641+1905346 & $\mathrm{b}$ \\
\hline Gaia & 661192902209491456 & $\mathrm{c}$ \\
\hline R.A. & 084006.42 & $\mathrm{c}$ \\
\hline Decl. & +190534.42 & $\mathrm{c}$ \\
\hline$\mu_{\text {R.A. }}\left(\right.$ mas $\left.\mathrm{yr}^{-1}\right)$ & $-15.92 \pm 0.12$ & $\mathrm{c}$ \\
\hline$\mu_{\mathrm{decl} .}\left({\left.\operatorname{mas~} \mathrm{yr}^{-1}\right)}^{-1}\right.$ & $-129.02 \pm 0.07$ & $\mathrm{c}$ \\
\hline Parallax (mas) & $12.582 \pm 0.075$ & $\mathrm{c}$ \\
\hline $\mathrm{RV}\left(\mathrm{km} \mathrm{s}^{-1}\right)$ & $40.6 \pm 0.2$ & This work \\
\hline Spectral type & $\mathrm{M} 3.0 \mathrm{~V}$ & d \\
\hline$T_{\text {eff }}(\mathrm{K})$ & $3385 \pm 70$ & d \\
\hline$[\mathrm{Fe} / \mathrm{H}](\mathrm{dex})$ & $-0.02 \pm 0.12$ & d \\
\hline $\log g$ & $4.906 \pm 0.041$ & d \\
\hline$M \mathrm{~s}\left(M_{\odot}\right)$ & $0.358 \pm 0.042$ & d \\
\hline$R \mathrm{~s}\left(R_{\odot}\right)$ & $0.350 \pm 0.035$ & d \\
\hline$L s\left(L_{\odot}\right)$ & $0.015 \pm 0.003$ & d \\
\hline$v \sin i\left(\mathrm{~km} \mathrm{~s}^{-1}\right)$ & $<2.00$ & This work \\
\hline \multicolumn{3}{|c|}{ Photometric Magnitudes } \\
\hline Kep & 15.03 & $\mathrm{a}$ \\
\hline Gaia $G$ & 14.98 & $\mathrm{c}$ \\
\hline Johnson $B$ & 17.69 & e \\
\hline Johnson $V$ & 16.18 & $\mathrm{e}$ \\
\hline$J$ & 12.18 & $\mathrm{~b}$ \\
\hline$H$ & 11.60 & $\mathrm{~b}$ \\
\hline$K$ & 11.37 & $\mathrm{~b}$ \\
\hline
\end{tabular}

Note. References for the sources: (a) EXOFOP-K2: https://exofop.ipac.caltech. edu/k2/; (b) The Two Micron All Sky Survey (2MASS; Skrutskie et al. 2006); (c) Gaia DR2 (Gaia Collaboration et al. 2016, 2018); (d) Hirano et al. (2018); (e) The AAVSO Photometric All-sky Survey (APASS; Henden et al. 2009).

relative motion of the star), to the data. To avoid influence from time-dependant variability, we simultaneously obtained a third order polynomial fit over the time. This fit was then applied to the whole segment, including the transits, to correct for possible correlation with the telescope pointing. After the correlation to the POS_CORR columns have been removed, we masked outliers and transit events again before fitting a seventh order polynomial to the light curve segments to correct for stellar variability. Finally, all light curve segments are normalized and stacked together.

We compared light curves generated from our custom pipeline with ones that are publicly available from the Vanderburg \& Johnson (2014) pipeline (K2SFF) and from Luger et al. (2018) (EVEREST; kindly provided by Luger). We found that the noise levels of the light curves from the three pipelines are comparable. Light curves from Campaign 16 and Campaign 18 generated from our pipeline have a slightly lower overall scatter, whereas the scatter in the Campaign 5 light curve is slightly higher than that in the K2SFF pipeline. For a consistent analysis, we opted to use the light curves obtained from our pipeline (as shown in Figure 1) for light-curve modeling and TTV analysis.

\subsection{Planet Detection}

We searched the $K 2$ light curves for transit signals using the DST algorithm (Cabrera et al. 2012), which optimizes the fit to the transit shapes with a parabolic function. Figure 2 shows the periodograms of the DST statistics measured in all light curves. The top left panel of Figure 2 shows that the $\sim 2.6$ day signal of $\mathrm{K} 2-146 \mathrm{~b}$ was detected in $\mathrm{C} 05$, and subsequently in $\mathrm{C} 16$ and $\mathrm{C} 18$.

The 2.6 day signal was then filtered and the light curves were analyzed with the DST algorithm again. Strong peaks at $\sim 4$ days are found in the periodograms of the C16 and C18 data, as shown in the bottom left panel of Figure 2. However, no significant detection is found in $\mathrm{C05}$, and transits of the outer planet were not observed upon visual inspection due to the noise level of the C05 light curve. We also ran our transit search algorithm on the $\mathrm{K} 2 \mathrm{SFF} \mathrm{C} 05$ data since it has a slightly lower scatter. Although we detected hints of a transit signal at $\sim 4$ days, the detection was not significant. We attribute this to a precessing orbital plane of this outer planet, which we discuss in later sections.

The characterization of the multi-planet system K2-146 follows the approach outlined here: the transit parameters are derived iteratively. We first performed a global analysis to extract the transit times and transit parameters of planet $b$ and planet c (Section 4). The transit parameters of the two planets were analyzed independently using a stacked transit light curve. We then model the transit times of the planets to derive their respective orbital elements (Section 5). Finally, we use information from the TTV-deduced orbital elements to model the stacked transits of planet $\mathrm{b}$ and planet $\mathrm{c}$, and improve the precision of the system parameters (Section 6).

\section{HPF Observations}

We obtained spectra with the Habitable-zone Planet Finder (HPF) between 2019 February 22 and 2019 May 9. HPF is a highresolution $(R=55,000)$ precision-fiber-fed, near-infrared (NIR) spectrograph recently installed on the Hobby-Eberly Telescope, covering the information-rich $z, Y$, and $J$ bands $(810-1280 \mathrm{~nm})$ (Mahadevan et al. 2012). HPF has demonstrated $\sim 1.53 \mathrm{~m} \mathrm{~s}^{-1} \mathrm{RV}$ precision on the sky in the NIR on the nearby bright M-dwarf star Barnard's star (Metcalf et al. 2019). The HPF echellograms are extracted into 1D spectra using the custom pipeline described in Kaplan et al. (2018) and Ninan et al. (2018).

\subsection{Absolute RV and Projected Rotational Velocities}

To measure the absolute and and projected rotational velocities of K2-146, we use the empirical spectral matching routines described in Stefansson et al. (2020), which closely follow the SpecMatch-emp algorithm described in Yee et al. (2017), adapted for use on a high signal-to-noise ratio $(\mathrm{S} / \mathrm{N})$ library of as-observed HPF spectra. Briefly, the spectral matching algorithm compares an as-observed spectrum of the target star to a library of high $\mathrm{S} / \mathrm{N}$ slowly rotating as-observed spectra with known stellar parameters using a $\chi^{2}$ figure of merit. The algorithm first aligns the target and library spectra in wavelength by accounting for the barycentric shift of the target star. The absolute RV of the target star is calculated and accounted for by cross-correlating the target spectrum by a binary mask as described in Stefansson et al. (2020). In doing so, we obtain an absolute RV value of $\gamma=40.6 \pm 0.2 \mathrm{~km} \mathrm{~s}^{-1}$, where the scatter is obtained by calculating the scatter of the absolute RV independently determined for 8 different HPF orders clean of tellurics.

After aligning the target and library spectra in wavelength, the spectra are resampled to a common wavelength grid. 


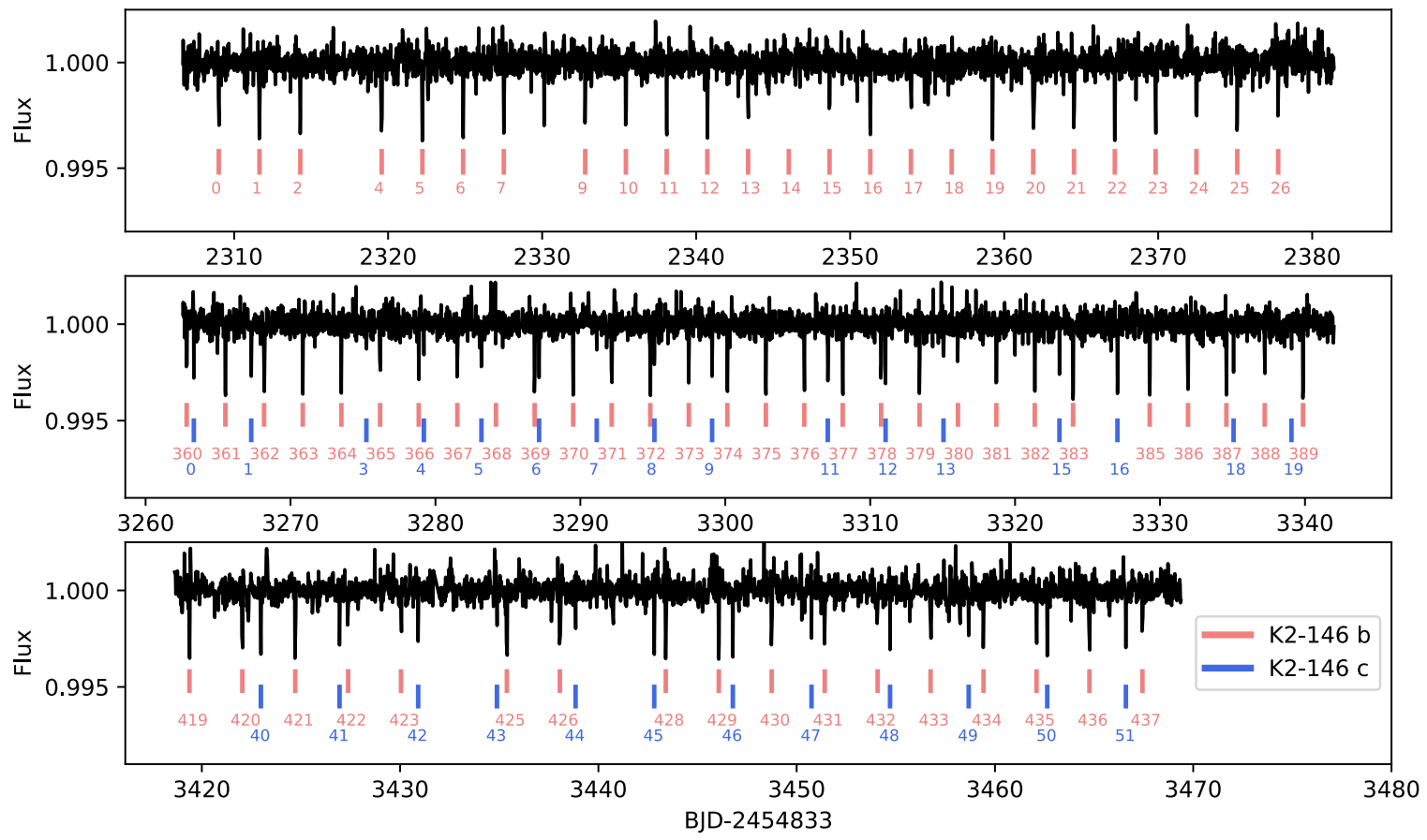

Figure 1. $K 2$ light curve of K2-146 from Campaigns 5 (top panel), Campaign 16 (middle panel), and Campaign 18 (bottom panel). The red and blue lines indicate transits of K2-146 b and K2-146 c used in transit modeling and TTV analysis. The numbers below the lines correspond to the integer transit epoch number from when the first transit became visible.

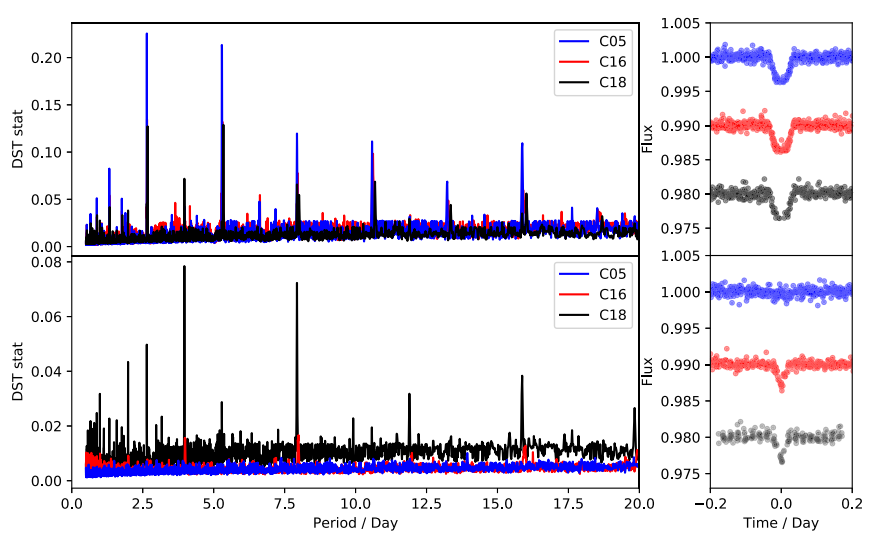

Figure 2. Top left: periodogram of the DST statistics evaluated as in Cabrera et al. (2012) for K2-146. We detect K2-146 b with the strongest peak at $\sim 2.6$ days. Top right: phase-folded light curves of K2-146 with TTV correction. The light curves are arbitrarily shifted for clarity. Bottom left: periodogram of the DST statistics after the signal of the inner planet is filtered. Bottom right: filtered, phase-folded light curves of K2-146 with TTV correction. The light curves are arbitrarily shifted for clarity. The transits of the outer planet were not significantly detected in $\mathrm{C} 05$, which we attribute to possible nodal precession of the orbital planet. The C05 light curve is phase-folded with the ephemeris derived from our transit search algorithm.

Residuals and a corresponding $\chi^{2}$ value are then calculated by subtracting from the target spectrum an artificially broadened version of the library spectrum that is also scaled by a fifthorder Chebychev polynomial to account for any residual systematics. A best-fit $v$ sin $i$ value for each library spectrum is then obtained by minimizing the $\chi^{2}$. This optimization is repeated for all of the library stars discussed in Stefansson et al. (2020). After this loop, the top five best-fit spectra are then further optimized to create a final composite spectrum that results in an even better fit to the observed target spectrum.
To arrive at a final $v \sin i$ value, and its associated scatter, we independently estimated the $v \sin i$ for five HPF orders clean of tellurics, resulting in five independent point estimates of the $v \sin i$. In doing so, we formally obtained a median value of $v \sin i=$ $1.1 \mathrm{~km} \mathrm{~s}^{-1}$ with an rms scatter of $200 \mathrm{~m} \mathrm{~s}^{-1}$. However, as discussed in Stefansson et al. (2020), this value is at the lower limit we estimate to be able to reliable measure the $v \sin i$ of the star, given the resolution of HPF of $R=55,000$, corresponding to $\mathrm{FWHM}=6 \mathrm{~km} \mathrm{~s}^{-1}$. Even though the derived values are all consistent at the $200 \mathrm{~m} \mathrm{~s}^{-1}$ level, at this resolution, we estimate that we should be able to detect rotational velocities upwards of $\sim 2 \mathrm{~km} \mathrm{~s}^{-1}$. We thus conclude that K2-146 has a $v \sin i<$ $2 \mathrm{~km} \mathrm{~s}^{-1}$ from the HPF spectra.

\subsection{RV Analysis}

Precise RVs are extracted from the HPF spectra via the method described in Metcalf et al. (2019) and Stefansson et al. (2020). Briefly, our RV extraction code is a modified version of SERVAL (Zechmeister et al. 2018), which determines RV by template-matching (using a $\chi^{2}$ metric) the stellar spectrum at a single epoch with a "master" template made by coadding all spectra of the target. This method has proven especially useful for M dwarfs (Anglada-Escudé \& Butler 2012), whose complex spectra are not as amenable to cross-correlation with a binary mask (e.g., Baranne et al. 1996) as hotter stars. The RV measurements of K2-146 are shown in Figure 3 and the values are listed in Appendix A.

We used the Lomb-Scargle (LS) periodogram (Lomb 1976; Scargle 1982) by implementing the fast algorithm of Press \& Rybicki (1989) to search for possible periodic signals in the RV data. The resulting power spectrum is shown in the upper right panel of Figure 3. We computed the corresponding false-alarmprobabilities (FAP) using the approximation method proposed by Baluev (2008), and found no significant peaks with a FAP 

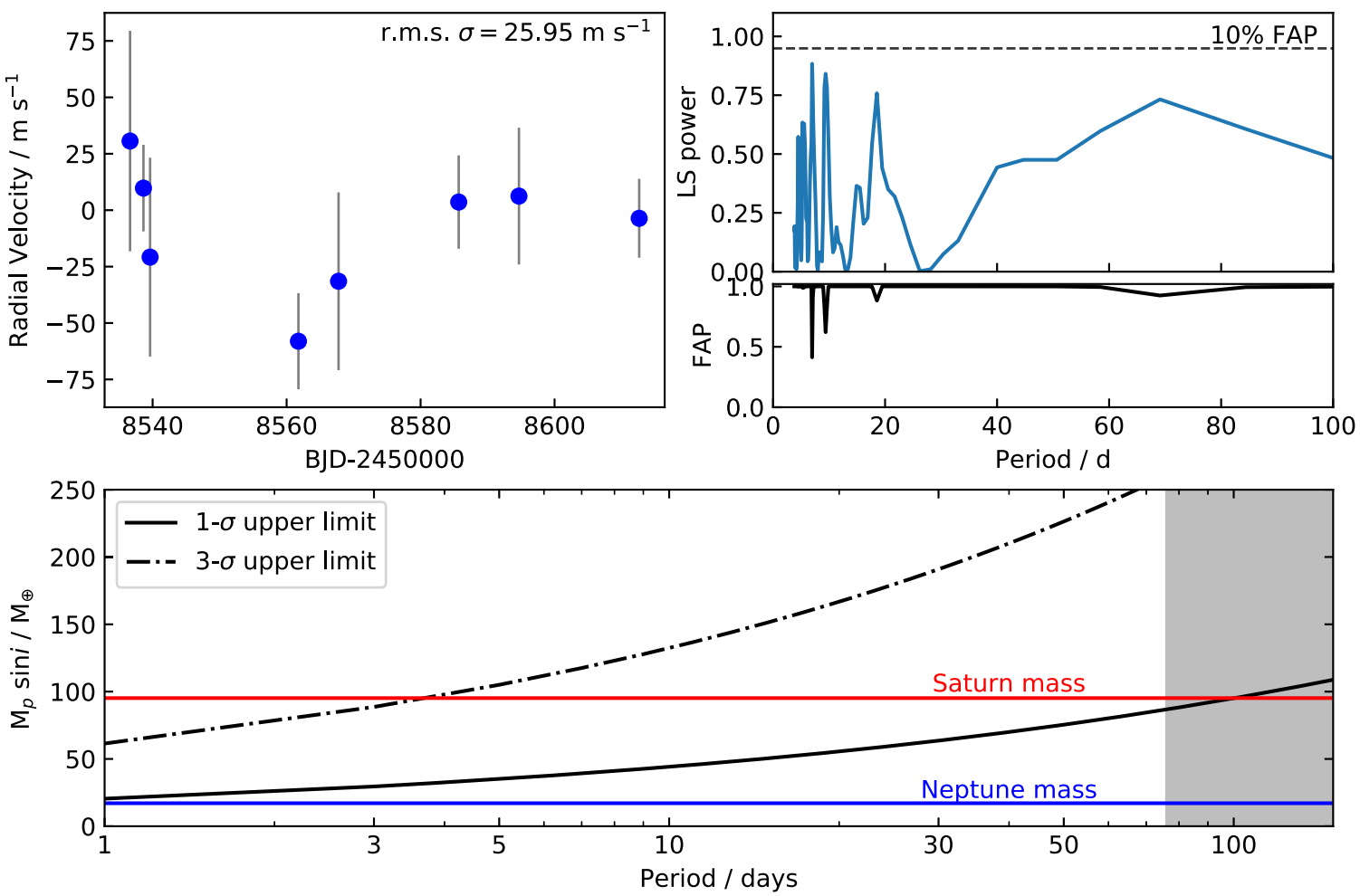

Figure 3. Radial velocity analysis of the HPF data. Top left: RV measurements for K2-146. The r.m.s of the data is $25.95 \mathrm{~m} \mathrm{~s}^{-1}$ and the mean uncertainty is $30.16 \mathrm{~m} \mathrm{~s}^{-1}$. Top right: Lomb-Scargle periodogram of the RV data for K2-146. The upper panel shows the power spectrum and the dashed line shows the level required for a maximum peak false-alarm probability (FAP) of 10\%. The bottom panel shows the corresponding FAP at different periods. Bottom: upper mass limits for planets around K2-146 calculated from the HPF RVs. The $1 \sigma$ and $3 \sigma$ upper mass limits of a planet in circular orbit are denoted by the solid and dashed lines, respectively. The gray shaded region shows the period beyond the time baseline of our observation. For $P<76$ days, we can rule out the presence of an outer planet with mass $M_{p} \sin i>86 M_{\oplus}$ (i.e., approximately Saturn mass) in a circular orbit.

of less than $10 \%$. The rms of the RV data is $\sigma=25.95 \mathrm{~m} \mathrm{~s}^{-1}$. This value was used as the $1 \sigma$ upper limit in the RV semiamplitude, $K$. We determined the upper mass limit $\left(M_{p} \sin i\right)$ of a possible outer planet by computing $M_{p} \sin i K / P$ over a range of periods $(P)$. The bottom panel of Figure 3 shows the $1 \sigma$ and $3 \sigma$ mass upper limits for an outer planet in a circular orbit. We are able to exclude the presence of an outer planet with $M_{p} \sin i>86 M_{\oplus}$ in a circular orbit for periods up to $\sim 76$ days (or $a<0.25 \mathrm{au}$ ).

\section{Transit Time Extraction}

In this section, we describe the method used to extract the times of K2-146 b and c. Two independent methods were implemented in our transit analysis to obtain the transit parameters. The final set of transit parameters will be refined following our TTV analysis.

\subsection{PyTTV}

We extracted the transit times using the Python Tool for Transit Variations (PyTTV; J. Korth 2020, in preparation). This tool uses PyTransit (Parviainen 2015) for transit modeling, $\mathrm{PyDE}^{37}$ for optimization and emcee (Foreman-Mackey et al. 2013) for posterior sampling.

Before modeling, the light curves around each transit were detrended by subtracting a second order polynomial fit to the out-of-transit light curve. These cutout segments were then the input for the detailed modeling with PyTTV. Some transits

\footnotetext{
${ }^{37}$ https://github.com/hpparvi/PyDE
}

were excluded from the analysis (see Figure 1): transit numbers $3,8,384,424$, and 427 for planet $\mathrm{b}$ and transit numbers 2,10 , 14 , and 17 for planet c. ${ }^{38}$

The transit time extraction and transit fits were performed for each planet independently. The individual transits are fitted collectively with the Mandel \& Agol (2002) model, each with their own transit center but sharing the rest of the transit parameters (individual transit fitting models are found in Figures B.3 and B.4). The optimization was done by computing the $\log$-likelihood, $\log L$, in our code to estimate transit parameters:

$$
\log L=-\frac{1}{2} \sum_{i=0}^{N}\left[\frac{\left(x_{i}-\mu_{i}\right)^{2}}{\sigma_{i}^{2}}+\ln \left(2 \pi \sigma_{i}^{2}\right)\right]
$$

where $x_{i}$ is the model, $\mu_{i}$ is the data, and $\sigma_{i}$ is the error in the data for the $i$ th point, respectively. The fitted parameters are radius ratio $k$, impact parameter $b$, and stellar density $\rho_{s}$, all with uniform priors. We used a quadratic limb-darkening model with the "triangular sampling" parameterization presented by Kipping (2013). Gaussian priors were imposed on the limb-darkening coefficients, $u_{1}$ and $u_{2}$, where the central

\footnotetext{
38 We checked the light-curve flags and found that transit numbers 3 and 424 both have flags 2048 (impulsive outlier) and 1048576 (thruster firing), transit number 8 has flag 8192 (cosmic ray), transit number 384 has flag 1024 (sudden sensitivity dropout), and transit number 427 has flag 32768 (no fine point). For planet $\mathrm{c}$ the transits with numbers 2 and 10 have flag 1048576. For transit number 14 and 17 we found no non-zero flags but they might not be visible because of the long-cadence observations (30 minutes) and are therefore missed in the light curve.
} 


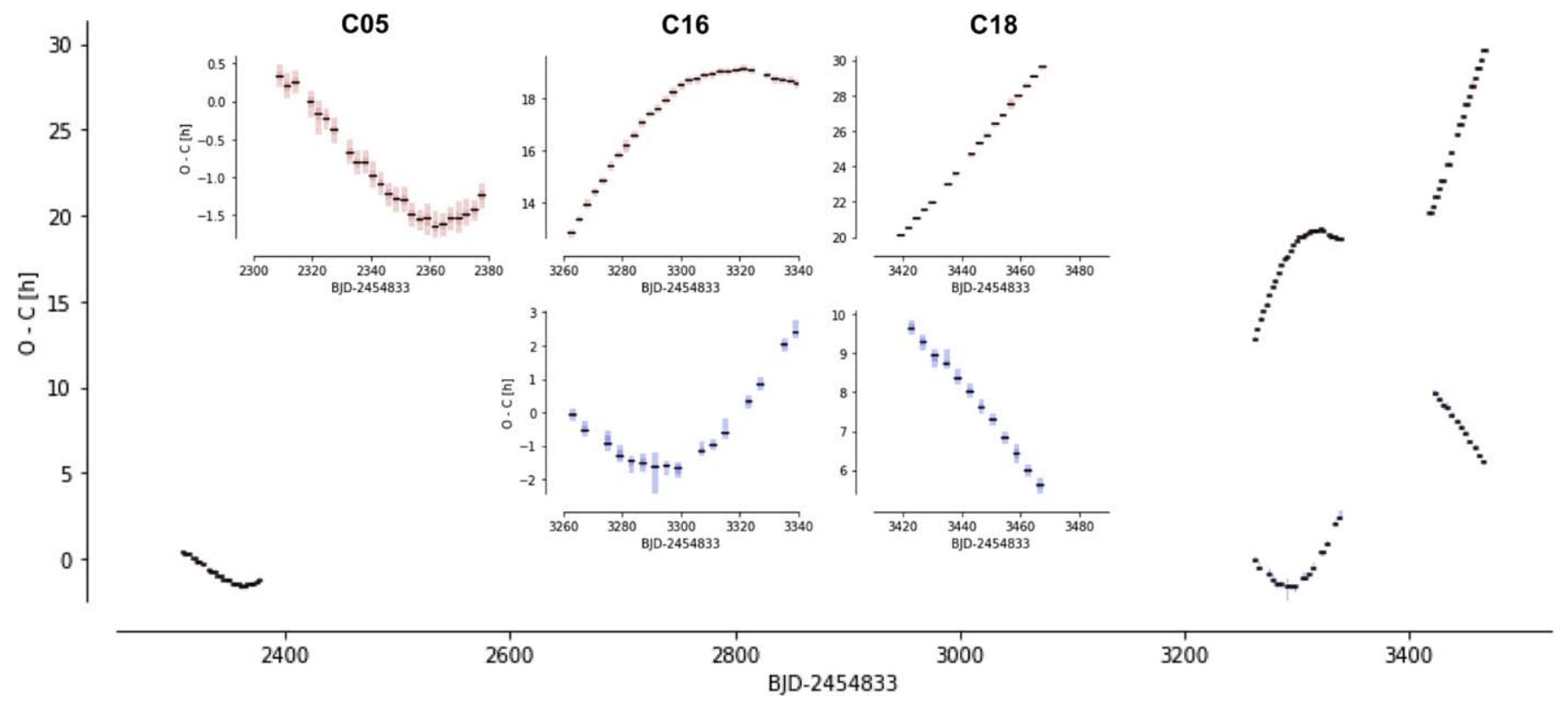

Figure 4. $O-C$ diagram of planet $\mathrm{b}$ (red) and planet c (blue). An anti-correlation in transit times is clearly visible in $\mathrm{C} 16$ and $\mathrm{C} 18$. The inset shows a magnification of individual campaigns. The colored error bars mark the percentiles of the fitted transit times.

values of the coefficients were calculated with PyLDTK (Parviainen \& Aigrain 2015), which utilizes the spectrum library of Husser et al. (2013). The transits of planet c is grazing, the $a / R_{s}$ and hence the mean stellar density is less well constraint. Thus the stellar density posterior from the planet $\mathrm{b}$ analysis was used as a stellar density prior in the planet $\mathrm{c}$ analysis. To account for the long exposure times of the $K 2$ observation we applied a supersampling $(n=10)$ as suggested in Kipping (2010).

For posterior sampling, we ran five MCMC chains with 5000 steps whereby the previous run was used as a burn-in for the current MCMC run. The chains were checked for convergence visually. The posteriors and the derived planetary parameters are shown in Appendix B. The resulting corner plots are shown in Figures B.1 and B.2. The transit times of both planets are used for a detailed TTV analysis in the following section.

The fitted transit times of planet $b$ (red) and planet c (blue) are shown in the $O-C$ diagram in Figure 4. An anti-correlation between the $O-C$ values is clearly visible in campaigns 16 and 18. This shows that the two planets are orbiting in the same system and that they are gravitationally interacting with each other, confirming the planetary nature of the signals. For a better visualization, the inset in Figure 4 shows a magnification of the different campaigns. The colored error bars mark the $1 \sigma$ and $3 \sigma$ uncertainties of the fitted transit times.

\subsection{Stacked Transit Analysis}

An independent transit analysis of K2-146 b and c was performed using stacked transit light curves. The light curves were cut such that only data within eight transit durations, centered on each transit, were used in our analysis. The selected transits of K2$146 \mathrm{~b}$ and K2-146 c used in our analysis are indicated by red and blue lines, respectively, in Figure 1.

We employed a Markov-chain Monte Carlo (MCMC) approach to derive the system parameters of K2-146. The stacked transits of K2-146 b and K2-146c were modeled using the analytical functions by Mandel \& Agol (2002). The transit model was implemented using the package PyTransit (Parviainen 2015), and a quadratic stellar limb-darkening law was applied. The fitted transit parameters are the planet-to-star radius ratios, $k_{\mathrm{b}}$ and $k_{\mathrm{c}}$, the orbital inclinations, $i_{\mathrm{b}}$ and $i_{\mathrm{c}}$, stellar density, $\rho_{s}$, and the triangle sampling (Kipping 2010) of the quadratic stellar limb-darkening coefficients $u_{1}$ and $u_{2}$, where uniform priors were used. The orbital periods of the planets are kept fixed.

The MCMC method was implemented using the Python package emcee (Foreman-Mackey et al. 2013) for Bayesian parameter estimation. $\chi^{2}$ statistics was used for likelihood estimation in our model. We computed the log-likelihood, log $L$, following Equation (1). An initial burn-in phase of 20 MCMC chains $\times 10,000$ steps was implemented to optimize the convergence of the fit. To obtain reasonable uncertainties in the transit parameters, we rescaled the error bars such that the value of the reduced $\chi^{2}$ equals 1 . We then initiated 100 MCMC chains of $5 \times 10^{4}$ steps to sample the posterior space. We checked for convergence and discarded the first 2000 steps, then adopted the median, 16th, and 84th percentiles of the samples in the marginalized posterior distributions as the fitted values and their $1 \sigma$ uncertainties. The results of the fitted transit parameters of K2-146 b and K2-146 c are presented in Appendix B. The bestfitted transit parameters of K2-146 b and K2-146 c obtained here are generally consistent within $\sim 1 \sigma$ with those derived by PyTTV.

\section{TTV Analysis}

We modeled the TTVs for the two planets using the TTVFast code (Deck et al. 2014), considering Newtonian gravitational interactions between the host star and the two planets alone. For each planet, we fitted planet-to-star mass ratio, orbital period $P$, eccentricity, and argument of periastron parameterization ( $\sqrt{e} \cos \omega$ and $\sqrt{e} \sin \omega$, so that the uniform priors on these parameters correspond to the priors flat in $e$ and $\omega$ ), and time $t_{0}$ of inferior conjunction closest to the dynamical epoch $t_{\text {epoch }}($ BJD-2454833) $=3467.8$. The elements are osculating Jacobi elements defined at $t_{\text {epoch}}$, and the time 


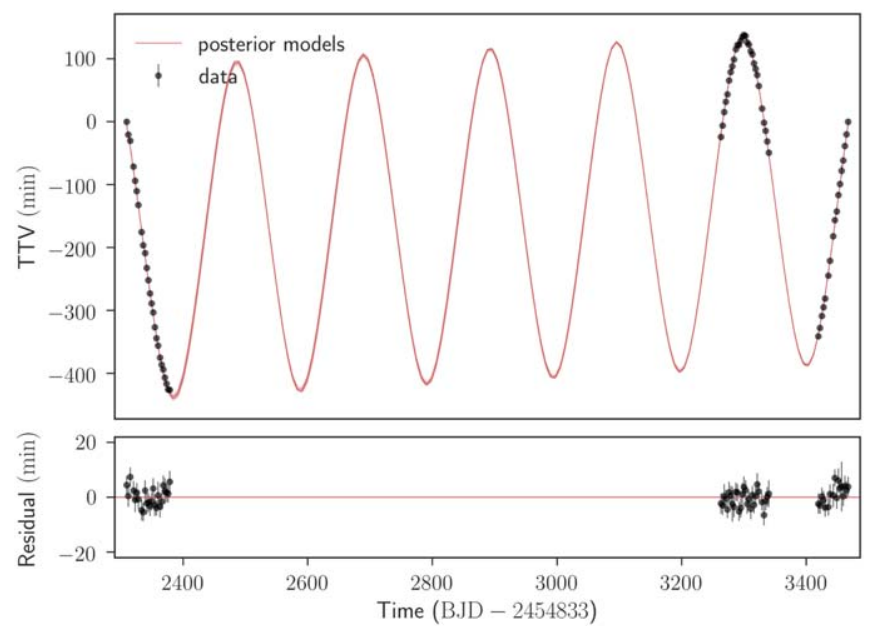

(a) K2-146 b

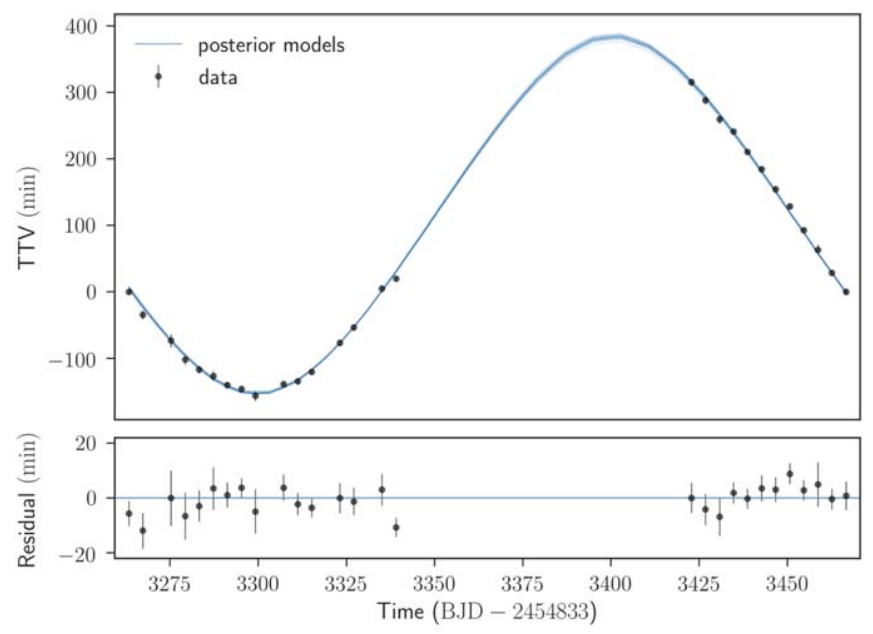

(b) $\mathrm{K} 2-146 \mathrm{c}$

Figure 5. Observed and modeled TTVs of K2-146 b (top) and c (bottom). Here TTVs are plotted with respect to the epoch BJD $=2454833+3467.4374$ and mean period 2.65696 days for planet $\mathrm{b}$ and; the epoch $\mathrm{BJD}=2454833+$ 3466.6029 and mean period 3.98579 days for planet $\mathrm{c}$. The thin blue lines are 20 random posterior models. The residuals are computed for the best-fit model.

$t_{0}$ is related to the time of periastron passage $\tau$ via $2 \pi\left(t_{0}-\tau\right) / P=$ $E_{0}-e \sin E_{0}, \quad$ where $\quad E_{0}=2 \arctan \left[\sqrt{\frac{1-e}{1+e}} \tan \left(\frac{\pi}{4}-\frac{\omega}{2}\right)\right]$. Considering that both planets are transiting in the C18 data, which are close to the dynamical epoch, the inclination and longitude of the ascending node at the epoch were fixed to be $\pi / 2$ and 0 , respectively. The likelihood was defined using the usual $\chi^{2}$ as $\exp \left(-\chi^{2} / 2\right)$, where we directly adopted the errors from PyTTV because the scatter in the data around the best model was found to be consistent with the assigned values. We adopted uniform priors for all these parameters and used emcee (Foreman-Mackey et al. 2013) to sample from their posterior distribution.

Figure 5 shows the TTV models generated with 20 sets of parameters randomly drawn from the posterior distribution. Table 2 summarizes the median and $68 \%$ credible interval of the marginal posterior distribution for each parameter: the upper parts show the fitted parameters, and the lower part
Table 2

Masses and Orbital Elements for K2-146 b and c Determined from TTV Modeling

\begin{tabular}{lcc}
\hline \hline & $\mathrm{K} 2-146 \mathrm{~b}$ & $\mathrm{~K} 2-146 \mathrm{c}$ \\
\hline Fitted Parameters & & \\
$M_{\mathrm{p}} / M_{\mathrm{s}}\left(\times 10^{-5}\right)$ & $2.7 \pm 0.2$ & $6.0 \pm 0.2$ \\
$P($ days $)$ & $-0.36_{-0.08}^{+0.11}$ & $3.9663 \pm 0.0002$ \\
$\sqrt{e} \cos \omega$ & $-0.07_{-0.08}^{+0.09}$ & $0.40_{-0.10}^{+0.08}$ \\
$\sqrt{e} \sin \omega$ & $3467.4345 \pm 0.0007$ & $0.01 \pm 0.07$ \\
$t_{0}(\mathrm{BJD}-2454833)$ & & $3466.6019 \pm 0.0009$ \\
Derived Parameters & $5.6 \pm 0.7$ & \\
$M_{\mathrm{p}}\left(M_{\oplus}\right)^{\mathrm{a}}$ & $0.14 \pm 0.07$ & $7.1 \pm 0.9$ \\
$e$ & $191_{-15}^{+11}$ & $0.16 \pm 0.07$ \\
$\omega($ deg $)$ & & $2_{-10}^{+12}$ \\
\hline
\end{tabular}

Notes. The values quoted here are the medians and symmetric $68 \%$ credible intervals of the marginal posteriors. The orbital elements are defined at the epoch $t_{\text {epoch }}(\mathrm{BJD}-2454833)=3467.8$.

${ }^{\mathrm{a}}$ Derived using $M_{\mathrm{s}}=0.358 \pm 0.042 M_{\odot}$.

shows the derived parameters. The planet-to-star mass ratios combined with the host star mass yield planetary masses of $5.6 \pm 0.7 M_{\oplus}$ for $\mathrm{K} 2-146 \mathrm{~b}$ and $7.1 \pm 0.9 M_{\oplus}$ for $\mathrm{K} 2-146 \mathrm{c}$. Moderate eccentricities are favored for both planets, but they show a strong negative correlation and one of the planets may have a nearly circular orbit. The $99.7 \%$ upper limit for the eccentricity is 0.3 for both planets. The observed difference in the arguments of periastron is consistent with anti-alignment of the apses. The implications of these features will be discussed in Section 7.

\section{Transit Modelling}

\subsection{Updating Transit Parameters}

The stacked transit light curves of K2-146 b and c were reanalyzed incorporating the eccentricity information from the TTV analysis. The transits were modeled with Transit and Light Curve Modeller (TLCM; Csizmadia 2020), a software tool for joint RV and transit light-curve fits, or transit light-curve fits only. It utilizes the Mandel \& Agol (2002) and Eastman et al. (2013) subroutines to calculate the transit light-curve shapes for every moment when we have an observation. A wavelet-filter (Carter \& Winn 2009) can be applied to model the red-noise effects. Contamination is also taken into account. The lightcurve part uses a quadratic limb-darkening law. The stellar radius, based on the value measured by Hirano et al. (2018), as well as the spectroscopic log $g$ values, can be used as priors for the fit. Parameter estimation is done via the Genetic Algorithm, refined by Simulated Annealing (Geem 2001). The final parameter estimation is done using several chains of MCMC with at least $10^{5}$ steps. The median and the width of the chains will define the final adopted solutions and their uncertainty ranges. The Gelman-Rubin statistic (e.g., Croll 2006) is used to check the convergence of chains. For detailed descriptions of TLCM, we refer the reader to the following works: Csizmadia et al. (2011, 2015), Smith et al. (2017).

The fitted parameters are the epoch of mid-transit, $T_{0}$, the scaled semimajor axis $\left(a / R_{s}\right)$, planet-to-stellar radius ratio $\left(R_{p} / R_{s}\right)$, the impact parameter, $b$, and the quadratic stellar limbdarkening coefficients $u_{+}=u_{1}+u_{2}$, and $u_{-}=u_{1}-u_{2}$. The orbital periods, $P$, of the planets were kept fixed. In addition, we used the eccentricity and argument of periastron derived from Section 5 as priors to perform our analysis. Table 3 
Table 3

Best-fit Planet Parameters of K2-146 b,c from a Stacked Transit Analysis, and Their Corresponding $1 \sigma$ Uncertainties

\begin{tabular}{llcc}
\hline \hline Parameter & Description (unit) & Values and Uncertainties \\
\hline$P$ & & K2-146 b & K2-146 c \\
$T_{0}$ & Period (day) & 2.6698 & 3.9663 \\
$R_{p}$ & Epoch (day from transit center) & $0.00009 \pm 0.00038$ & $-0.00015 \pm 0.00076$ \\
$a$ & Radius ( $R_{\oplus}$ ) & $2.25 \pm 0.10$ & $2.59_{-0.39}^{+1.81}$ \\
$b$ & Semimajor axis (au) & $0.0248 \pm 0.0002$ & $0.0327 \pm 0.0006$ \\
$i$ & Impact parameter & $0.391 \pm 0.069$ & $0.930 \pm 0.097$ \\
$R_{p} / R_{s}$ & Inclination ( ${ }^{\circ}$ & $88.5 \pm 0.3$ & $87.3 \pm 0.3$ \\
$a / R_{s}$ & Scaled planet radius & $0.0589 \pm 0.0014$ & $0.0680_{-0.0254}^{+0.1226}$ \\
$u_{+}=u_{1}+u_{2}$ & Scaled semimajor axis & $15.250 \pm 0.126$ & $20.064 \pm 0.412$ \\
$u_{-}=u_{1}-u_{2}$ & Combined limb-darkening coefficient & $0.575 \pm 0.171$ & $0.678 \pm 0.169$ \\
$\rho_{p}$ & Combined limb-darkening coefficient & $0.022 \pm 0197$ & $0.072 \pm 0.210$ \\
\hline
\end{tabular}

Note. The orbital periods $P$ of the planets were kept fixed at the values derived from the TTV analysis.

presents the best-fit transit parameters of $\mathrm{K} 2-146 \mathrm{~b}$ and K2-146 c. The resulting best-fit transit models of the inner and outer planets are shown in Figure 6.

\subsection{Planet Impact Parameters}

The outer planet K2-146 c was only found to transit in the latter campaigns $\mathrm{C} 16$ and $\mathrm{C} 18$. This suggests orbital plane precession is at play. We searched for seasonal changes in the impact parameters of both planets. The transits of the inner and outer planets were stacked separately in each $\mathrm{K} 2$ campaign. The mean orbital elements of each campaign were obtained from the posterior samples of the TTV analysis. We fixed $\sqrt{e} \sin \omega$ and $\sqrt{e} \cos \omega$ values on these mean values for the analysis of separate campaigns. We then ran TLCM on the campaign-stacked data; the free parameters in each campaign were the scaled semimajor axis, impact parameter, radius ratio, and epoch. The radius ratio was the same from campaign to campaign.

For planet $\mathrm{b}$, we obtained an impact parameter of $b=$ $0.42 \pm 0.11, b=0.25 \pm 0.10$, and $b=0.41 \pm 0.15$, for C05, $\mathrm{C} 16$, and $\mathrm{C} 18$ respectively. While the impact parameter is practically the same in $\mathrm{C} 05$ and $\mathrm{C} 18$, it differs by approximately $1 \sigma$ in $\mathrm{C} 16$ in comparison to the two other campaigns. This indicates that we do not see a significant change in the impact parameter of planet $b$ in these data. In the case of planet c, we obtained an impact parameter of $b=0.94 \pm 0.10$ and $b=0.92 \pm 0.10$ for C16 and C18, respectively. Again, we do not see significant changes in the impact parameter of the planet, although the null detection of transits in C05 does suggest that it has been drifting. We attribute this to a shorter time baseline between $\mathrm{C} 16$ and $\mathrm{C} 18(\sim 200$ days $)$ compared to the $\sim 1000$ day separation between C05 and C16.

\section{Discussion}

\subsection{Dynamic Stability of the K2-146 System}

We investigated the dynamical stability for the K2-146 multi-planet system, independent of the TTV analysis, to obtain the mass limits on the two Neptune-size planets and to establish the stability of the system.

\subsubsection{Hill Radius of K2-146}

A Hill-sphere is the region where the planet's gravity is dominating over the central star. The planets' mutual Hill radius is

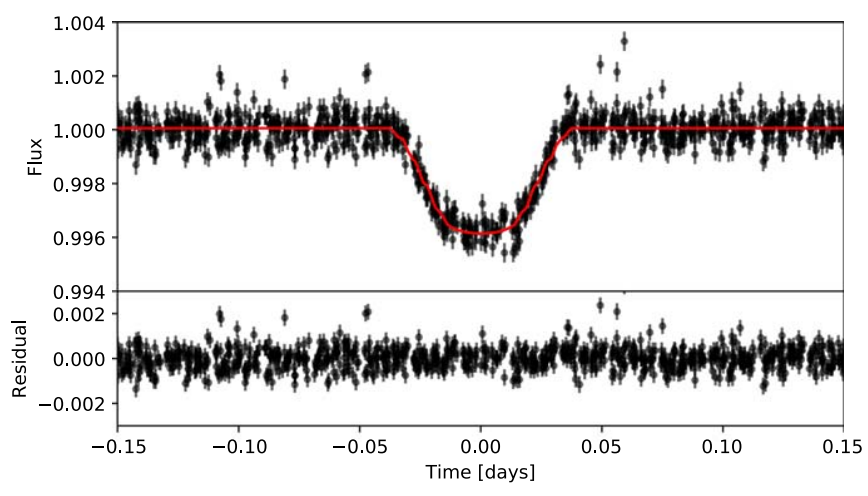

(a) K2-146 b

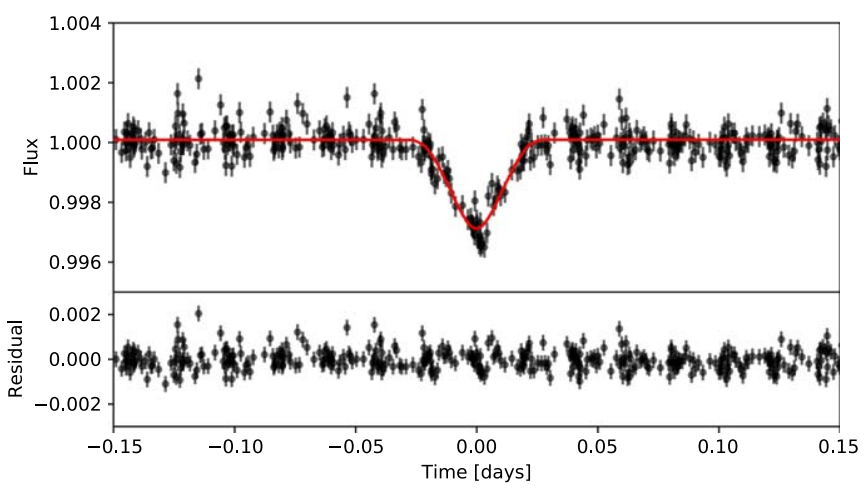

(b) K2-146 c

Figure 6. Stacked light curves showing the TTV-corrected transits of (a) K2146 b, and (b) K2-146 c in the top panels. The red lines are the best-fit transit model and the corresponding residuals are shown in the bottom panels.

defined as $R_{\mathrm{H}}=a_{1}\left(\left(m_{1}+m_{2}\right) / 3 M_{s}\right)^{1 / 3}$, where $m_{1}, m_{2}, M_{s}$ are the masses of the inner and outer planets and the host star, respectively, and $a_{1}$ is the semimajor axis of the inner planet. If the initial separation between two planets on an initially circular orbits, measured in Hill radii $\Delta_{\mathrm{H}}=\left(a_{2}-a_{1}\right) / R_{\mathrm{H}}$, is greater than $2 \sqrt{3} \sim 3.5$, then the system is Hill stable (Gladman 1993). The mutual separation in Hill radii in the case of K2-146 b,c is $\Delta_{\mathrm{H}}=9.67$. This indicates that the system is Hill stable and will never undergo close encounters. For planets on eccentric orbits, 
we refer readers to Marchal \& Bozis (1982) for the general threebody problem:.

\subsubsection{N-body Simulations of K2-146}

The $N$-body simulation code Mercury6 (Chambers 1999) was used to study the orbital evolution and determine the dynamic stability of the system. We chose the "hybrid symplectic and Bulirsch-Stoer integrator" mode of Mercury 6 to compute close encounters in the system. We adopted the stellar mass and radius reported in Hirano et al. (2018) for the central star.

We performed some initial simulations to place upper mass limits on the two planets based on their dynamic stability. We employed 5105 numerical integrations, each with an integration period of $2 \mathrm{Myr}$. An initial step size of 2.7 days was selected; subsequent step sizes were adjusted by the variable time-step algorithms in the program to maintain integration accuracy. The orbital parameters of the system were recorded every 2 yr. For each integration, the orbital periods $\left(P_{\mathrm{b}}\right.$ and $\left.P_{\mathrm{c}}\right)$ were chosen from a Gaussian distribution using the center and $1 \sigma$ reported by PyTTV in Section 4. The eccentricities of the planets $\left(e_{\mathrm{b}}\right.$ and $\left.e_{\mathrm{c}}\right)$ were drawn from a uniform distribution between $e=0$ and $e=0.3$. The two planets were assumed to be coplanar with fixed inclinations and longitudes of the ascending node where $i_{\mathrm{b}}$, $i_{\mathrm{c}}=90^{\circ}$ and $\Omega_{\mathrm{b}}, \Omega_{\mathrm{c}}=0^{\circ}$. The arguments of periastron $\left(\omega_{\mathrm{b}}\right.$ and $\omega_{c}$ ) and mean anomalies of the planets were drawn from a uniform sample where $0^{\circ}<\omega_{\mathrm{b}}, \omega_{\mathrm{c}}<360^{\circ}$ and $0^{\circ}<\mathcal{M}_{\mathrm{b}}, \mathcal{M}_{\mathrm{c}}<360^{\circ}$. The planetary masses of K2-146 b and K2-146 c $\left(M_{\mathrm{b}}\right.$ and $M_{\mathrm{c}}$ respectively) were randomly drawn from a half-normal distribution with a width of $1000 M_{\oplus}$. In each simulation, the system becomes unstable when (1) the planets collide with one another or with the central star; or (2) the planets are ejected from the system, i.e., $e_{\mathrm{b}}, e_{\mathrm{c}}>1$ and/or $a>10$ au. Our results showed that a significant fraction of systems are dynamically stable for $2 \mathrm{Myr}$ for planets with masses up to $\sim 30 M_{\oplus}$. We found that over $90 \%$ of these systems remained stable for $2 \mathrm{Myr}$ if the mass ratio of $\mathrm{K} 2-146 \mathrm{~b}$ and $\mathrm{K} 2-146 \mathrm{c}$ is close to unity.

We further used a subset of the posterior samples from our TTV analysis in Section 5 to check the stability of the system. We performed 153 simulations where the starting orbital parameters of the system were defined by randomly selecting posterior samples from the TTV analysis. Again, each simulation was integrated for a period of $2 \mathrm{Myr}$, the step sizes were automatically adjusted by the algorithm, and the orbital parameters were recorded for every 2 yr. All 153 of our simulations were shown to be stable over at least 2 Myr. Figure 7 shows the planet masses drawn from our TTV posterior sample.

\subsection{Orbital Resonance of the Sub-Neptunes}

The orbital periods of K2-146 b and K2-146 c show a 3:2 commensurability. To assess whether the planet pair is trapped in a 3:2 MMR, we monitored the orbital evolution simulation of the so-called resonance angles over a $2000 \mathrm{yr}$ long interval.

The resonance arguments, $\Theta_{1}$ and $\Theta_{2}$, for a pair of planets in a 3:2 MMR are defined as

$$
\begin{aligned}
& \Theta_{1}=3 \cdot \lambda_{\mathrm{c}}-2 \cdot \lambda_{\mathrm{b}}-\varpi_{\mathrm{b}}, \\
& \Theta_{2}=3 \cdot \lambda_{\mathrm{c}}-2 \cdot \lambda_{\mathrm{b}}-\varpi_{\mathrm{c}} .
\end{aligned}
$$

The quantity $\lambda=\mathcal{M}+\varpi$ is often called the mean longitude, and $\mathcal{M}$ is the mean anomaly. The longitude of pericenter is $\varpi=\omega+\Omega$ (e.g., Murray \& Dermott 1999). The angle between two planets at conjunction is $0^{\circ}$. One can think of

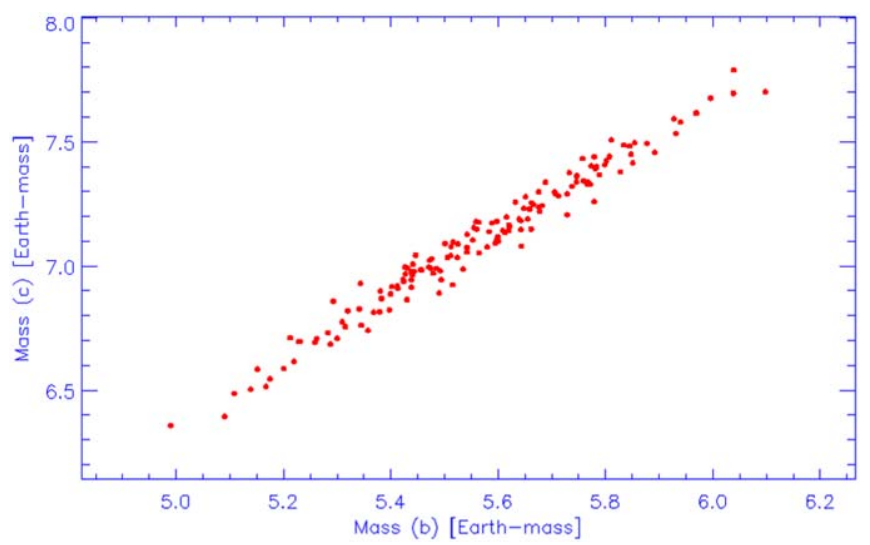

Figure 7. Mass-mass plot of all numerical simulations of the multi-planet system K2-146. The masses of K2-146 b and K2-146 c were randomly drawn from our TTV posterior samples. We showed that all 153 simulations are stable for at least 2 Myr.

each resonant angle as tracking the angle at which conjunctions happen, relative to each planet's pericenter (e.g., when $\lambda_{\mathrm{c}}=\lambda_{\mathrm{b}}$, the conjunction occurs at $\lambda_{\mathrm{b}}-\varpi_{\mathrm{b}}=\Theta_{1}$ ).

We performed 20,000 simulations to evaluate the resonance angles of the planet pair. In each simulation, the planet-to-star mass ratios and orbital elements of the planets were drawn from the posterior samples of the TTV analysis. The stellar mass in Table 1 was used to convert the mass ratios into planetary masses. Then the orbits were numerically integrated for $2000 \mathrm{yr}$. The values of the relevant parameters were saved for every 36 days of the integration, then the resonant angles were calculated. For each integration, we recorded the maximum amplitude of the resonant angles. We took the modulo $360^{\circ}$ values of the resonant variables.

Figure 8 shows the histograms of the maximum halfamplitude of the resonant angles. The wide peak at around $\Theta_{1} / 2=135^{\circ}$ is because the location of conjunctions librates with a half-amplitude of $\approx 135^{\circ}$ around a location roughly antialigned with the pericenter of planet b's orbit. We found approximately $74 \%$ of simulations show libration with a halfamplitude of around $135^{\circ}$. Figure 9 shows the simulation that gave the smallest libration angle. For the remaining cases, the resonance angles either always circulate, or chaotically switch from libration to circulation. The special cases can be tested in more detailed dynamics studies in the future.

The widths of the peaks in these histograms are caused by the uncertainties of the masses and orbital elements derived from TTVs. To increase their precisions, more transit observations are needed from this system. Despite the faintness of the host star, which leads to lower accuracy in TTVs, it would be worthwhile to try to observe it with high cadence with Transiting Exoplanet Survey Satellite (TESS; Ricker et al. 2014) and PLAnetary Transits and Oscillations (PLATO of stars; Rauer et al. 2014). Alternatively, observations may also be obtained with the less precise ( $\sim 10$ minutes) timing values from CHaracterising ExOPlanet Satellite (CHEOPS; Broeg et al. 2013), because the precision could be compensated by many hours of TTV-values in this case.

\subsection{Planet Composition}

Our TTV analysis in Section 5 and transit parameters refined in Section 6 revealed that K2-146 b has a mass and radius of $2.25 \pm 0.10 R_{\oplus}$ and $5.6 \pm 0.7 M_{\oplus}$, respectively, 


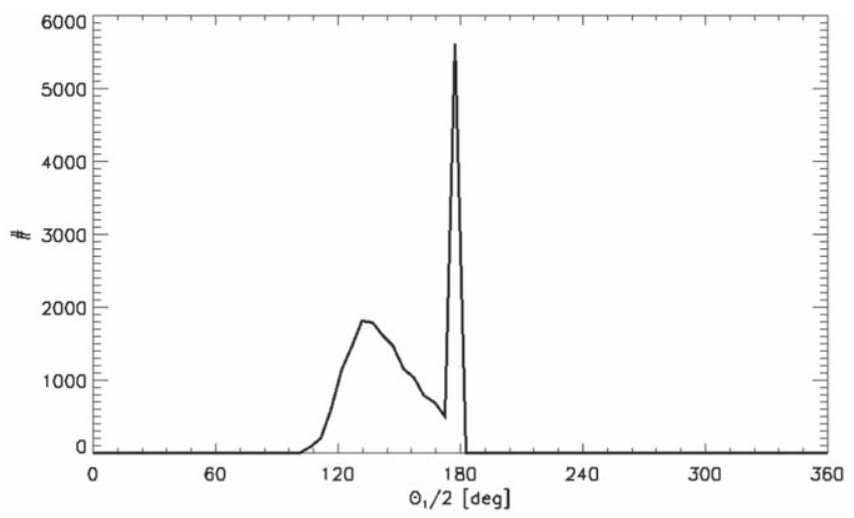

(a) Resonance angle $\Theta_{1}=3 \lambda_{c}-2 \lambda_{b}-\varpi_{b}$

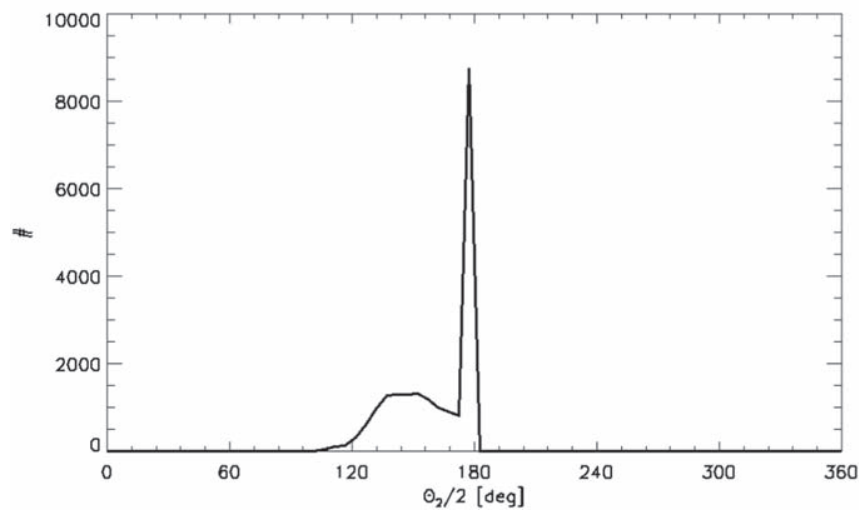

(b) Resonance angle $\Theta_{2}=3 \lambda_{c}-2 \lambda_{b}-\varpi_{c}$

Figure 8. The histograms of the half-maximum amplitude of the resonant angles $\Theta_{1}$ (top) and $\Theta_{2}$ (bottom). We find that $74 \%$ of the 20,000 simulations with orbital elements drawn from the TTV posterior samples have $\Theta_{1} \sim 135^{\circ}$. This suggests the locations of the conjunction librates with a half-amplitude of $\approx 135^{\circ}$.

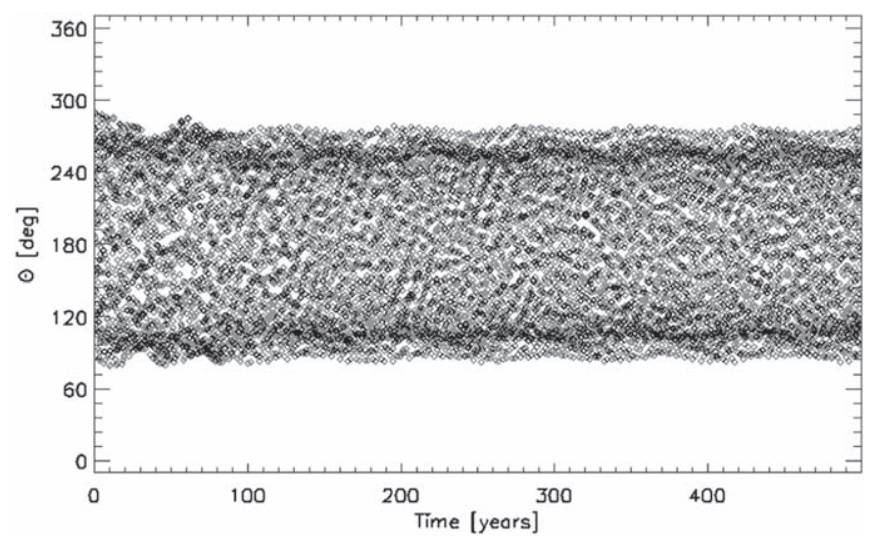

Figure 9. Evolution of the orbital solution giving the smallest libration halfamplitude. This variability of $\Theta_{1}$ means that the location of the conjunctions librates with a half-amplitude of $\approx 110^{\circ}$ around a location roughly anti-aligned with the pericenter of planet b's orbit.

corresponding to a bulk density of $2.702 \pm 0.494 \mathrm{~g} \mathrm{~cm}^{-3}$. The mass and radius of the outer planet K2-146 c are $2.59_{-0.39}^{+1.81} R_{\oplus}$ and $7.1 \pm 0.9 M_{\oplus}$, respectively, which correspond to a bulk density of $2.246_{-1.846}^{+1.883} \mathrm{~g} \mathrm{~cm}^{-3}$. K2-146 b and K2-146 c are orbiting at distances of 0.0248 au and 0.0327 au, respectively.

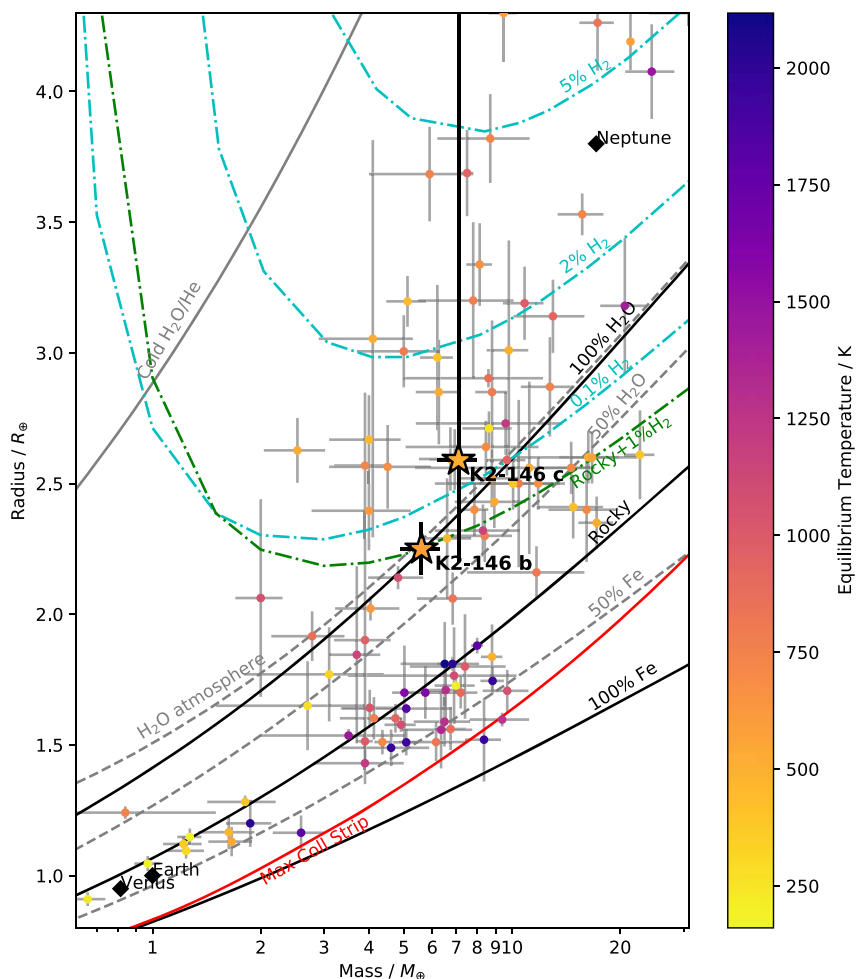

Figure 10. Mass-radius plot of known planets with masses constrained to a precision of better than approximately $30 \%$. The masses and radii of K2-146 b and $\mathrm{c}$ are indicated by the stars. The colors of each data point show the planet equilibrium temperature as indicated by the color bar on the right. The massradius relations of small planets of different compositions are taken from Zeng et al. $(2016,2019)$. The different compositions are indicated by the solid $(100 \%$ water, $100 \%$ rock, or $100 \%$ iron), dashed (mixtures of water, rock and iron), and dashed-dotted lines (water-rich cores with a hydrogen envelope or Earthlike rocky cores with a hydrogen envelope). Solar system planets are labeled with black diamonds. The red solid line gives the minimum radii of a rocky planets constraint from a giant impact model (Marcus et al. 2010).

We assumed the planets have an albedo of 0 , and a reradiation factor of one-fourth, where atmospheric circulation redistributes the energy around the planetary atmosphere, then reradiates the energy back into space. Under these assumptions, the equilibrium temperatures of planet $\mathrm{b}$ and planet $\mathrm{c}$ are approximately $590 \mathrm{~K}$ and $520 \mathrm{~K}$, respectively.

Figure 10 shows a mass-radius plot of known planets with $M_{p}<30 M_{\oplus}$ where the mass of the planets are determined with a precision better than approximately $30 \%$. The solid, dashed, and dashed dot lines represents the mass-radius relations for different planetary compositions as derived in Zeng et al. (2016, 2019). The color of each data point indicates a planet's equilibrium temperature corresponding to the color bar. The masses and radii of K2-146 b and c are consistent with cases of a $100 \% \mathrm{H}_{2} \mathrm{O}$ interior, a water-rich core with the addition of an $\mathrm{H}_{2} \mathrm{O}$-gaseous atmosphere, or an Earth-like rocky interior with a small fraction of $\mathrm{H}_{2}$ envelope. The large radius uncertainty of $\mathrm{K} 2-146 \mathrm{c}$ means that it could have a more massive $\mathrm{H}_{2}$ envelope.

\subsection{Formation and Evolution}

Observational evidence of planet orbital architectures allows one to place constraints on the formation and dynamical evolution of the system. In the case of K2-146, we observed a number of interesting traits: 
1. Masses and radii of the planets are consistent with a water-dominated core and the presence of water or an $\mathrm{H}_{2}$ envelope-it was previously proposed that in situ formation of mini Neptunes and super Earths is possible if $50-100 M_{\oplus}$ of rocky material is delivered to the inner disk for planet assembly (Hansen \& Murray 2012). However, the large fraction of solids would drift toward the host star on a relatively short timescale, preventing in situ formation. Furthermore, in situ formation is unlikely to produce a significant fraction of atmospheric masses for close-in planets (Schlichting 2014; Inamdar \& Schlichting 2015). Therefore, close-in planets with an atmosphere were likely formed at larger separation from the star in the presence of a gas disk. Subsequently, planets accrete gaseous envelopes as they migrate inward toward their current locations.

2. The evolution of resonance arguments for the planet pair suggests that K2-146 $b$ and $c$ are likely trapped in a 3:2 mean motion resonance-during formation, planets interact with protoplanetary disks. This drives the migration of planets inward through the disk due to exchange of angular momentum (Goldreich \& Tremaine 1979, 1980; Lin \& Papaloizou 1979). Convergent migration can occur in one of two ways: (1) planets formed at wide separations can move toward one another with different migration speeds; (2) planets formed in close proximity are massive enough to form a gap in the disk where inner and outer disks push the planets toward each other. When the planet orbital periods approach a commensurability, dynamical interactions that follow cause planets to migrate collectively inward while preserving period commensurability (Snellgrove et al. 2001). Under favorable disk parameters, planet masses, and migration speeds, the planet pair can enter a 3:2 MMR after breaking the 2:1 MMR barrier (e.g., Kley 2000; Nelson \& Papaloizou 2002), such as the case of HD 45364 (Correia et al. 2009) and KOI-1599 (Panichi et al. 2019).

3. Our TTV model revealed that both the inner and outer planets have moderate eccentricities of $e_{b}=0.14 \pm 0.07$ and $\mathrm{e}_{\mathrm{b}}=0.16 \pm 0.07$, and are apsidally anti-aligned, i.e., $\Delta \omega=$ $\omega_{\mathrm{b}}-\omega_{\mathrm{c}} \approx 180^{\circ}$ - convergent migration could have played a role in the observed eccentricity in the K2-146 planet pair. After the planets are captured in resonance, the planet pair migrates inward while maintaining resonance, which leads to orbital eccentricity excitation (Lee \& Peale 2002; Batygin \& Morbidelli 2013). While the protoplanetary disk is present, the planets could experience eccentricity damping as they migrate. The moderate eccentricities observed in K2-146 b and c imply that the migration process must be fast enough in order to minimize the damping efficiency. The anti-aligned apsides are a natural result of such a migration process. The conjunctions occur when K2-146 c is near periapse and K2-146 b is near apoapse. The longitudes of periapse of both planets are required to precess at the same rate for a stable configuration, such that the lines of apsides are locked in an anti-aligned state (Lee \& Peale 2002). Due to the close proximity of the two planets, such a mechanism is present to avoid close encounters.

4. The outer planet K2-146c showed a change in impact parameter-gravitational interaction between planets can give rise to apsidal and nodal precession around the host star. The change in impact parameter of the outer planet observed from K2 Campaign 5 to Campaign 16 is likely an indication of orbital precession. A misalignment between the planet orbits can be suspected, resulting in a precession of the line of nodes of planet c (Miralda-Escudé 2002). The precession would then lead to a change in the length of the transit chord. In our TTV analysis, we assumed the planets have coplanar orbits because, if the mutual inclination is large, the two planets are unlikely to transit simultaneously even if their orbits are precessing (see Mills \& Fabrycky 2017). This assumption could be tested directly via a joint modeling of TTVs and TDVs, or a photodynamical model of the light curves, which will enable a measurement of the mutual orbital inclination through the constraint on the nodal precession rate (e.g., Kepler-117 Almenara et al. 2015, Kepler-108 Mills \& Fabrycky 2017).

\section{Summary and Conclusion}

The strong TTV initially detected in the mini-Neptune K2$146 \mathrm{~b}$ suggested the presence of an additional body in the system. Further photometric observations from $K 2$ revealed an additional mini-Neptune K2-146 c orbiting at a 4 day period, forming a 3:2 MMR with the inner planet. The long observation baseline allowed precise determination of the planet masses via TTV. This demonstrates the importance of follow-up transit observations for parameter and dynamical constraints of a TTV system.

$N$-body simulations of K2-146 performed in this work provided a glimpse into the possible stability and resonance configuration of the planet pair. We found that the planets are probably captured into a 3:2 MMR during migration, and that their current orbital configuration can be dynamically stable for at least $2 \mathrm{Myr}$. Furthermore, the change in the impact parameter of the outer planet suggests some orbital plane precession, resulting in the displacement of the chord of transit and hence the change in transit depth and duration of K2-146 c. This effect can be further investigated using both TTVs and TDVs to constrain the orbit precession rate, and mutual inclination in the system. Further observations with TESS, and in the future PLATO can also provide a better precision in the transit times measurement. A detailed migration model would be valuable to study different precession rates leading to a stable orbital configuration.

Small planets around $\mathrm{M}$ dwarfs are frequently found in multi-planet systems. In fact, occurrence studies suggest that there are typically around 2.5 small planets $\left(R_{p}<4 R_{\oplus}\right)$ per M dwarf with periods shorter than 200 days (Dressing \& Charbonneau 2015). Only a small handful of planets are known around $\mathrm{M}$ dwarfs that have planetary radii and masses below the radius and mass of Neptune (e.g., TRAPPIST-1 system; Gillon et al. 2017, LHS-1140 system; Dittmann et al. 2017; Ment et al. 2019, L 98-59 System; Kostov et al. 2019, Gl 357 system; Luque et al. 2019). These are laboratories to test planet formation theories and dynamical evolutions, providing clues to the processes involved in building multi-planet systems containing the smallest possible planets.

The expected RV semi-amplitudes of K2-146 b and K2$146 \mathrm{c}$ are $K_{\mathrm{b}}=5.1 \mathrm{~m} \mathrm{~s}^{-1}$ and $K_{\mathrm{c}}=5.7 \mathrm{~m} \mathrm{~s}^{-1}$. Our RV followups using HPF were able to rule out the presence of an outer massive companion up to around $0.25 \mathrm{au}$. They also demonstrated that mass determination of the sub-Neptunes K2-146 b and $\mathrm{c}$ using RV data can be challenging for such a faint host star $(J=12.18 \mathrm{mag})$. In order to achieve the precision required to detect the RV signals of sub-Neptune mass planets around faint $\mathrm{M}$ dwarfs, future $30 \mathrm{~m}$ class telescopes may need to be equipped with high-resolution spectrographs (e.g., European Extremely Large Telescope (E-ELT); Udry et al. 2014). 
We thank our referee, Dan Tamayo, for insightful feedback which improved the manuscript. We thank Mareike Godolt for helpful discussion of interpreting planet compositions. K.W.F.L., J.K., Sz.Cs., M.E., S.G., A.P.H., M.P., and H.R. acknowledge support by D.F.G. grants PA525/18-1, PA525/19-1, PA525/20-1, HA3279/12-1, and RA714/14-1 within the D.F.G. Schwerpunkt SPP 1992, "Exploring the Diversity of Extrasolar Planets." Sz.Cs. thanks the Hungarian National Research, Development and Innovation Office for the NKFI-KH-130372 grant. M.F., I.G., and C.M.P. gratefully acknowledge the support of the Swedish National Space Agency (DNR 163/16 and 174/18). This work is partly supported by JSPS KAKENHI grants No. JP18H01265 and JP18H05439, and JST PRESTO grant No. JPMJPR1775. O.B. acknowledges support from the UK Science and Technology Facilities Council (STFC) under grants ST/S000488/1 and ST/R004846/1. S.M. acknowledges support from the Spanish Ministry under the Ramon y Cajal fellowship number RYC-201517697. P.K. acknowledges support by GACR 17-01752J. S.A. and E.K. acknowledge support by the Danish Council for Independent Research, through a DFF Sapere Aude Starting Grantnr. 4181-00487B. S.A., E.K., and M.L. acknowledge support by the Stellar Astrophysics Centre, for which funding is provided by The Danish National Research Foundation (Grant agreement No.: DNRF106). This work was supported by the KESPRINT collaboration, an international consortium devoted to the characterization and research of exoplanets discovered with space-based missions. www.kesprint.science. This work has made use of data from the European Space Agency (ESA) mission Gaia (https:// www.cosmos.esa.int/gaia), processed by the Gaia Data Processing and Analysis Consortium (DPAC, https://www.cosmos.esa.int/ web/gaia/dpac/consortium). Funding for the DPAC has been provided by national institutions, in particular the institutions
Table A.1

Radial Velocities of K2-146 from HPF

\begin{tabular}{lcc}
\hline \hline Time (BJD-2450000) & RV $\left(\mathrm{m} \mathrm{s}^{-1}\right)$ & Error $\left(\mathrm{m} \mathrm{s}^{-1}\right)$ \\
\hline 2458536.632234 & 71.6485 & 48.8470 \\
2458538.631308 & 50.7917 & 19.1782 \\
2458539.623356 & 20.2161 & 44.0408 \\
2458561.764655 & -17.0513 & 21.3058 \\
2458567.755665 & 9.5267 & 39.4031 \\
2458585.691166 & 44.6155 & 20.6901 \\
2458594.680752 & 47.2499 & 30.3059 \\
2458612.634185 & 37.4150 & 17.4908 \\
\hline
\end{tabular}

participating in the Gaia Multilateral Agreement. This research has made use of NASA's Astrophysics Data System Bibliographic Services, the ArXiv preprint service hosted by Cornell University.

\section{Appendix A}

Radial Velocity Measurements from HPF Precise RVs of K2146 were measured using the HPF to check for possible massive outer companions. The RV measurements are listed in Table A.1.

\section{Appendix B}

Supplementary Figures and Best-fit Transit Model from PyTTV The transit times of K2-146 b and c were fitted using PyTTV (J. Korth 2020, in preparation). Figures B.1 and B.2 show corner plots of the posterior distributions of the transit parameters for K2-146 b and c, respectively. The best-fit transit model and transit times of individual transits of K2-146 b and $c$ are shown in Figures B.3 and B.4, respectively. The best-fit transit parameters derived by PyTTV are listed in Table B.1. The results of the 


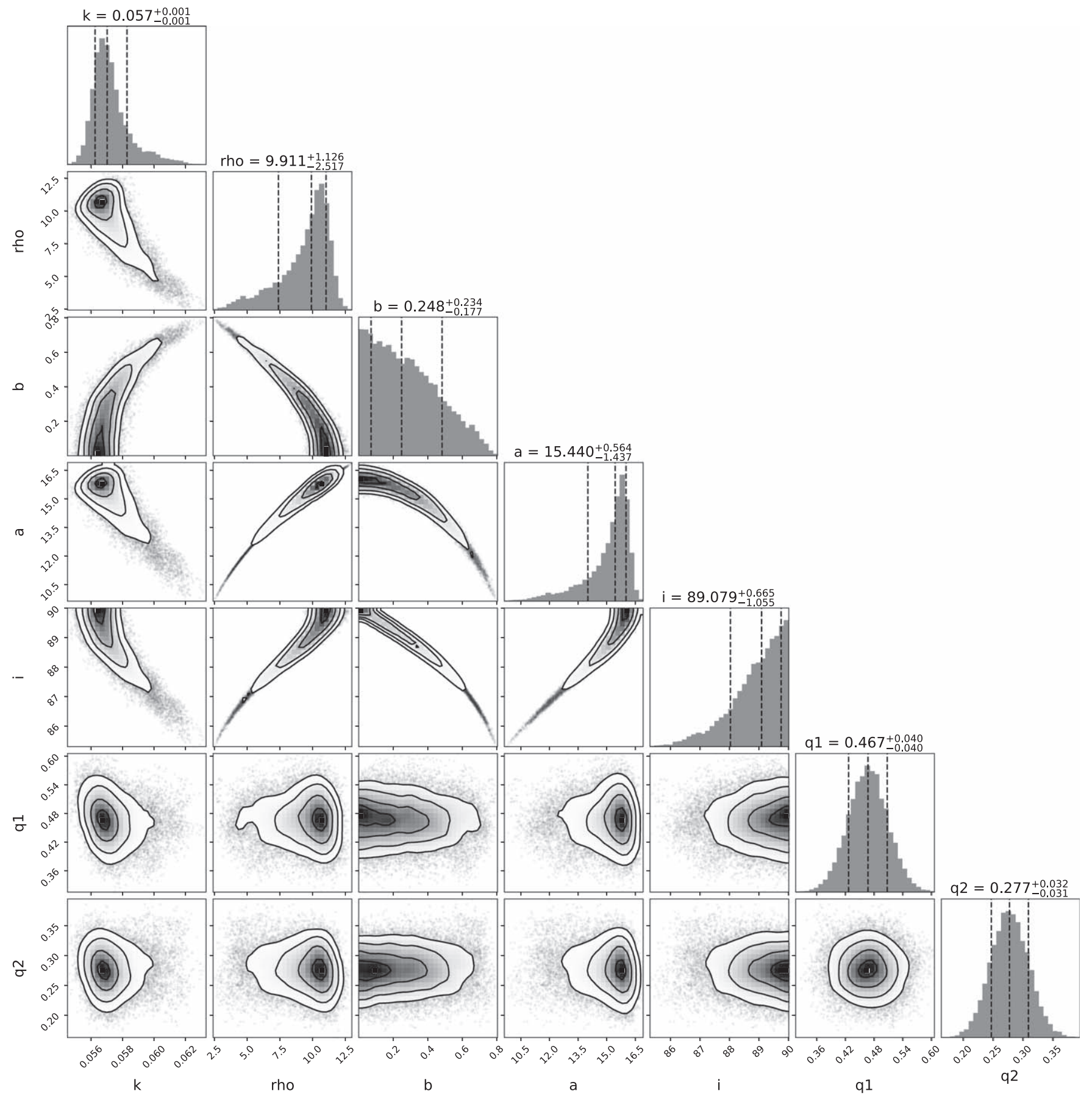

Figure B.1. Corner plot of the posterior distributions of the transit parameters for K2-146 b. The values above each column are the means of the posterior distributions with their respective $1 \sigma$ uncertainties. Note that the impact paramter is not well-constrained. 


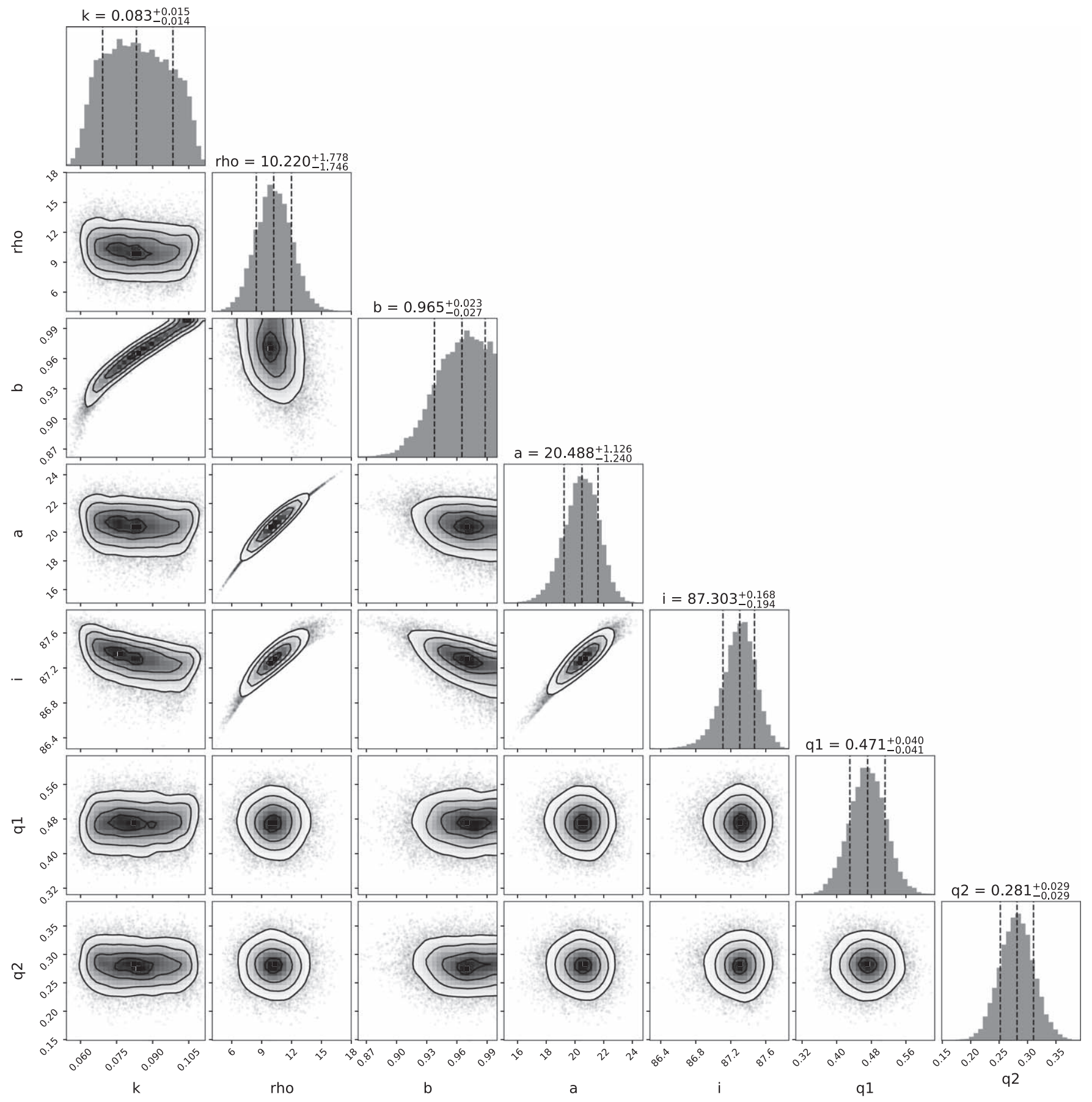

Figure B.2. Corner plot of the posterior distributions of the transit parameters for K2-146 c. The values above each column are the means of the posterior distributions with their respective $1 \sigma$ uncertainties. Note that the impact parameter is not well-constrained. 


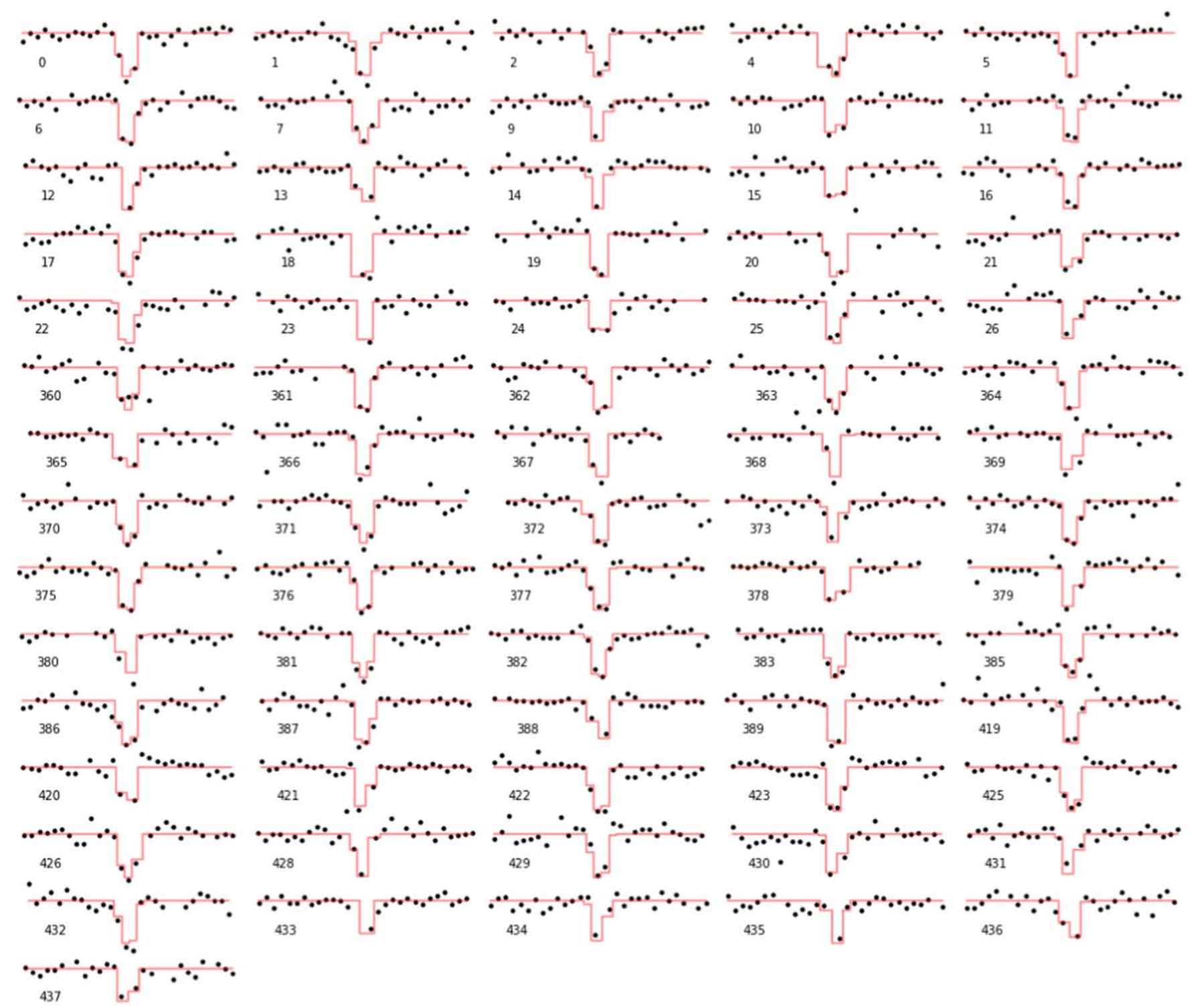

Figure B.3. Individual transits of K2-146 b fitted in the PYTTV transit parameter analysis. The $K 2$ data are denoted by the black points and the red solid lines are the best-fit transit models. The numbers in the bottom left corner of each transit plot correspond to the transit epoch number labeled in Figure 1. The individual transit times are available as data behind the Figure.

(The data used to create this figure are available.) 


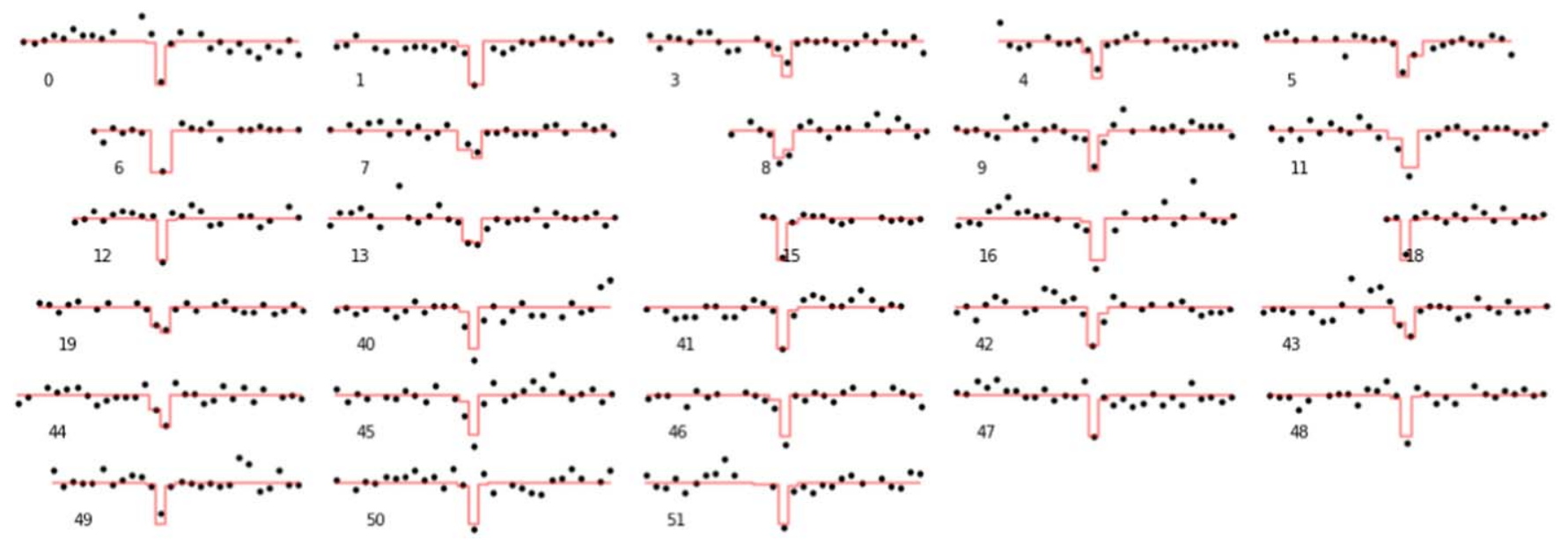

Figure B.4. Individual transits of K2-146 c fitted in the PyTTV transit parameter analysis. This figure uses the same notations as Figure B.3. The individual transit times are available as data behind the Figure.

(The data used to create this figure are available.)

Table B.1

Transit Parameters of K2-146 b and K2-146 c Derived from PyTTV Analysis and Stacked Transit Analysis

\begin{tabular}{|c|c|c|c|c|}
\hline \multirow{2}{*}{ Parameter } & \multicolumn{2}{|c|}{ PyTtV } & \multicolumn{2}{|c|}{ Stacked Transit Analysis } \\
\hline & $\mathrm{K} 2-146 \mathrm{~b}$ & K2-146 c & $\mathrm{K} 2-146 \mathrm{~b}$ & K2-146 c \\
\hline$P$ (day) & $2.65044 \pm 0.00007$ & $3.98974 \pm 0.00069$ & $\ldots$ & $\ldots$ \\
\hline$b$ & $0.27 \pm 0.19$ & $0.96 \pm 0.02$ & $0.03 \pm 0.03$ & $0.81 \pm 0.03$ \\
\hline$a / R_{\mathrm{S}}$ & $15.06 \pm 1.16$ & $20.44 \pm 1.19$ & $15.51 \pm 0.03$ & $20.38 \pm 0.07$ \\
\hline$i\left({ }^{\circ}\right)$ & $88.9 \pm 0.9$ & $87.3 \pm 0.2$ & $89.9 \pm 0.11$ & $87.7 \pm 0.09$ \\
\hline$a(\mathrm{au})$ & $0.0246 \pm 0.0031$ & $0.0332 \pm 0.0039$ & $0.0252 \pm 0.0166$ & $0.0332 \pm 0.0001$ \\
\hline
\end{tabular}

Note. The reported posterior values reported are the median and $1 \sigma$ uncertainties.

independent transit analysis on the stacked transit light curves of K2-146 b and c are also shown in Table B.1.

\section{ORCID iDs}

Kristine W. F. Lam (iD https://orcid.org/0000-0002-9910-6088 Kento Masuda (10 https://orcid.org/0000-0003-1298-9699 Philipp Eigmüller (i) https:// orcid.org/0000-0003-4096-0594 Gumundur Kári Stefánsson (ib) https://orcid.org/0000-00017409-5688

Michael Endl (iD https://orcid.org/0000-0002-7714-6310

Paul Robertson (10) https://orcid.org/0000-0003-0149-9678

John H. Livingston (i) https://orcid.org/0000-0002-4881-3620

Teruyuki Hirano (1D https://orcid.org/0000-0003-3618-7535

Oscar Barragán (iD https://orcid.org/0000-0003-0563-0493

Juan Cabrera (ib https://orcid.org/0000-0001-6653-5487

William D. Cochran (iD https://orcid.org/0000-00019662-3496

Fei Dai (iD https://orcid.org/0000-0002-8958-0683

Hans J. Deeg (D) https://orcid.org/0000-0003-0047-4241

Malcolm Fridlund (iD https://orcid.org/0000-0003-2180-9936

Davide Gandolfi (i) https://orcid.org/0000-0001-8627-9628

Sascha Grziwa (D) https://orcid.org/0000-0003-3370-4058

Petr Kabath (ib https://orcid.org/0000-0002-1623-5352
Suvrath Mahadevan (iD https://orcid.org/0000-00019596-7983

Savita Mathur (1D https://orcid.org/0000-0002-0129-0316 Prajwal Niraula (iD https://orcid.org/0000-0002-8052-3893

Seth Redfield (i) https://orcid.org/0000-0003-3786-3486 Ignasi Ribas (D) https://orcid.org/0000-0002-6689-0312 Marek Skarka (1D https://orcid.org/0000-0002-7602-0046 Jan Subjak (1D https://orcid.org/0000-0002-5313-9722 Vincent Van Eylen (1D https://orcid.org/0000-0001-5542-8870

\section{References}

Agol, E., Steffen, J., Sari, R., \& Clarkson, W. 2005, MNRAS, 359, 567 Almenara, J. M., Díaz, R. F., Mardling, R., et al. 2015, MNRAS, 453, 2644 Anglada-Escudé, G., \& Butler, R. P. 2012, ApJS, 200, 15 Armstrong, D. J., Santerne, A., Veras, D., et al. 2015, A\&A, 582, A33 Baluev, R. V. 2008, MNRAS, 385, 1279

Baranne, A., Queloz, D., Mayor, M., et al. 1996, A\&AS, 119, 373

Barros, S. C. C., Almenara, J. M., Demangeon, O., et al. 2015, MNRAS, 454, 4267

Batalha, N. M., Rowe, J. F., Bryson, S. T., et al. 2013, ApJS, 204, 24 Batygin, K., \& Morbidelli, A. 2013, AJ, 145, 1

Borucki, W. J., Koch, D., Basri, G., et al. 2010, Sci, 327, 977

Borucki, W. J., Koch, D. G., Basri, G., et al. 2011, ApJ, 728, 117

Broeg, C., Fortier, A., Ehrenreich, D., et al. 2013, EPJWC, 47, 03005 
Cabrera, J., Csizmadia, S., Erikson, A., Rauer, H., \& Kirste, S. 2012, A\&A, 548, A44

Carter, J. A., Agol, E., Chaplin, W. J., et al. 2012, Sci, 337, 556

Carter, J. A., \& Winn, J. N. 2009, ApJ, 704, 51

Chambers, J. E. 1999, MNRAS, 304, 793

Correia, A. C. M., Udry, S., Mayor, M., et al. 2009, A\&A, 496, 521

Croll, B. 2006, PASP, 118, 1351

Csizmadia, S. 2020, MNRAS, accepted

Csizmadia, S., Hatzes, A., Gandolfi, D., et al. 2015, A\&A, 584, A13

Csizmadia, S., Moutou, C., Deleuil, M., et al. 2011, A\&A, 531, A41

Deck, K. M., Agol, E., Holman, M. J., \& Nesvorný, D. 2014, ApJ, 787, 132

Dittmann, J. A., Irwin, J. M., Charbonneau, D., et al. 2017, Natur, 544, 333

Dressing, C. D., \& Charbonneau, D. 2013, ApJ, 767, 95

Dressing, C. D., \& Charbonneau, D. 2015, ApJ, 807, 45

Dressing, C. D., Vanderburg, A., Schlieder, J. E., et al. 2017, AJ, 154, 207

Eastman, J., Gaudi, B. S., \& Agol, E. 2013, PASP, 125, 83

Fabrycky, D. C., Lissauer, J. J., Ragozzine, D., et al. 2014, ApJ, 790, 146

Foreman-Mackey, D., Hogg, D. W., Lang, D., \& Goodman, J. 2013, PASP, 125,306

Fressin, F., Torres, G., Charbonneau, D., et al. 2013, ApJ, 766, 81

Gaia Collaboration, Brown, A. G. A., Vallenari, A., et al. 2018, A\&A, 616, A1 Gaia Collaboration, Prusti, T., de Bruijne, J. H. J., et al. 2016, A\&A, 595, A1

Geem, Z. W., Kim, J. H., \& Loganathan, G. V. 2001, Simul, 76, 60

Gillon, M., Triaud, A. H. M. J., Demory, B.-O., et al. 2017, Natur, 542, 456 Gladman, B. 1993, Icar, 106, 247

Goldreich, P., \& Tremaine, S. 1979, ApJ, 233, 857

Goldreich, P., \& Tremaine, S. 1980, ApJ, 241, 425

Hansen, B. M. S., \& Murray, N. 2012, ApJ, 751, 158

Henden, A. A., Welch, D. L., Terrell, D., \& Levine, S. E. 2009, AAS Meeting, 214, 407.02

Hirano, T., Dai, F., Gandolfi, D., et al. 2018, AJ, 155, 127

Holman, M. J., \& Murray, N. W. 2005, Sci, 307, 1288

Howard, A. W., Marcy, G. W., Bryson, S. T., et al. 2012, ApJS, 201, 15

Howell, S. B., Sobeck, C., Haas, M., et al. 2014, PASP, 126, 398

Husser, T. O., Wende-von Berg, S., Dreizler, S., et al. 2013, A\&A, 553, A6

Inamdar, N. K., \& Schlichting, H. E. 2015, MNRAS, 448, 1751

Kaplan, K. F., Bender, C. F., Terrien, R., et al. 2018, in ASP Conf. Ser. 523, Astronomical Data Analysis Software and Systems XXVIII, ed.

P. J. Teuben et al. (San Fransisco, CA: ASP), 568

Kipping, D. M. 2010, MNRAS, 408, 1758

Kipping, D. M. 2013, MNRAS, 435, 2152

Kley, W. 2000, MNRAS, 313, L47

Kostov, V. B., Schlieder, J. E., Barclay, T., et al. 2019, AJ, 158, 32

Lee, M. H., \& Peale, S. J. 2002, ApJ, 567, 596

Libralato, M., Nardiello, D., Bedin, L. R., et al. 2016, MNRAS, 463, 1780

Lin, D. N. C., \& Papaloizou, J. 1979, MNRAS, 186, 799

Lissauer, J. J., Ragozzine, D., Fabrycky, D. C., et al. 2011, ApJS, 197, 8

Livingston, J. H., Crossfield, I. J. M., Petigura, E. A., et al. 2018, AJ, 156, 277

Lomb, N. R. 1976, Ap\&SS, 39, 447
Luger, R., Kruse, E., Foreman-Mackey, D., Agol, E., \& Saunders, N. 2018, AJ, 156, 99

Luque, R., Pallé, E., Kossakowski, D., et al. 2019, A\&A, 628, A39

Mahadevan, S., Ramsey, L., Bender, C., et al. 2012, Proc. SPIE, 8446, $84461 \mathrm{~S}$

Mandel, K., \& Agol, E. 2002, ApJL, 580, L171

Marchal, C., \& Bozis, G. 1982, CeMec, 26, 311

Marcus, R. A., Sasselov, D., Hernquist, L., \& Stewart, S. T. 2010, ApJL, 712, L73

Masuda, K. 2017, AJ, 154, 64

Ment, K., Dittmann, J. A., Astudillo-Defru, N., et al. 2019, AJ, 157, 32

Metcalf, A. J., Anderson, T., Bender, C. F., et al. 2019, Optic, 6, 233

Millholland, S., Wang, S., \& Laughlin, G. 2017, ApJL, 849, L33

Mills, S. M., \& Fabrycky, D. C. 2017, AJ, 153, 45

Miralda-Escudé, J. 2002, ApJ, 564, 1019

Murray, C. D., \& Dermott, S. F. 1999, Solar System Dynamics (Cambridge: Cambridge Univ. Press)

Narita, N., Hirano, T., Fukui, A., et al. 2015, ApJ, 815, 47

Nelson, R. P., \& Papaloizou, J. C. B. 2002, MNRAS, 333, L26

Nespral, D., Gandolfi, D., Deeg, H. J., et al. 2017, A\&A, 601, A128

Nesvorný, D., Kipping, D., Terrell, D., et al. 2013, ApJ, 777, 3

Nesvorný, D., Kipping, D. M., Buchhave, L. A., et al. 2012, Sci, 336, 1133

Ninan, J. P., Bender, C. F., Mahadevan, S., et al. 2018, Proc. SPIE, 10709, $107092 \mathrm{U}$

Panichi, F., Migaszewski, C., \& Goździewski, K. 2019, MNRAS, 485, 4601

Parviainen, H. 2015, MNRAS, 450, 3233

Parviainen, H., \& Aigrain, S. 2015, MNRAS, 453, 3821

Petigura, E. A., Howard, A. W., \& Marcy, G. W. 2013, PNAS, 110, 19273

Pope, B. J. S., Parviainen, H., \& Aigrain, S. 2016, MNRAS, 461, 3399

Press, W. H., \& Rybicki, G. B. 1989, ApJ, 338, 277

Rauer, H., Catala, C., Aerts, C., et al. 2014, ExA, 38, 249

Ricker, G. R., Winn, J. N., Vanderspek, R., et al. 2014, Proc. SPIE, 9143 914320

Scargle, J. D. 1982, ApJ, 263, 835

Schlichting, H. E. 2014, ApJL, 795, L15

Skrutskie, M. F., Cutri, R. M., Stiening, R., et al. 2006, AJ, 131, 1163

Smith, A. M. S., Gandolfi, D., Barragán, O., et al. 2017, MNRAS, 464 2708

Snellgrove, M. D., Papaloizou, J. C. B., \& Nelson, R. P. 2001, A\&A, 374, 1092

Stefansson, G., Cañas, C., \& Wisniewski, J. 2020, AJ, 159, 100

Udry, S., Lovis, C., Bouchy, F., et al. 2014, arXiv:1412.1048

Vanderburg, A., \& Johnson, J. A. 2014, PASP, 126, 948

Weiss, L. M., Marcy, G. W., Petigura, E. A., et al. 2018, AJ, 155, 48

Yee, S. W., Petigura, E. A., \& von Braun, K. 2017, ApJ, 836, 77

Zechmeister, M., Reiners, A., Amado, P. J., et al. 2018, A\&A, 609, A12

Zeng, L., Jacobsen, S. B., Sasselov, D. D., et al. 2019, PNAS, 116, 9723

Zeng, L., Sasselov, D. D., \& Jacobsen, S. B. 2016, ApJ, 819, 127 